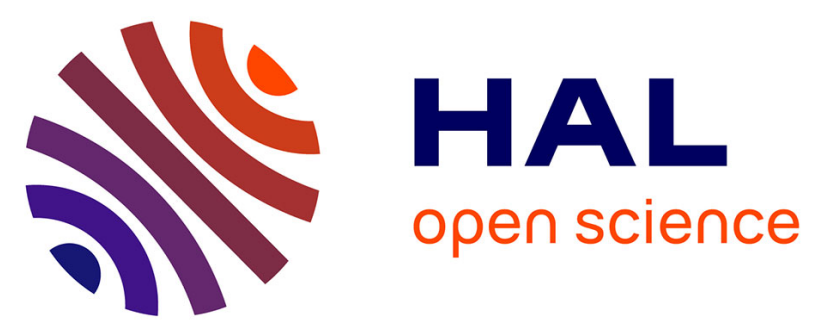

\title{
Piezoelectric nanoelectromechanical systems integrating microcontact printed lead zirconate titanate films
}

Daisuke Saya, Denis Dezest, Aaron Welsh, Fabrice Mathieu, Olivier Thomas, Thierry Leichle, Susan Trolier-Mckinstry, Liviu Nicu

\section{- To cite this version:}

Daisuke Saya, Denis Dezest, Aaron Welsh, Fabrice Mathieu, Olivier Thomas, et al.. Piezoelectric nanoelectromechanical systems integrating microcontact printed lead zirconate titanate films. Journal of Micromechanics and Microengineering, 2020, 30 (3), pp.035004. 10.1088/1361-6439/ab60bf . hal$02453806 \mathrm{v} 2$

\section{HAL Id: hal-02453806 \\ https://hal.science/hal-02453806v2}

Submitted on 16 Nov 2020

HAL is a multi-disciplinary open access archive for the deposit and dissemination of scientific research documents, whether they are published or not. The documents may come from teaching and research institutions in France or abroad, or from public or private research centers.
L'archive ouverte pluridisciplinaire HAL, est destinée au dépôt et à la diffusion de documents scientifiques de niveau recherche, publiés ou non, émanant des établissements d'enseignement et de recherche français ou étrangers, des laboratoires publics ou privés. 


\title{
Piezoelectric nanoelectromechanical systems integrating microcontact printed PZT films
}

\author{
Daisuke Saya ${ }^{1}$, Denis Dezest ${ }^{1}$, Aaron J. Welsh ${ }^{2}$, Fabrice Mathieu $^{1}$, Olivier Thomas ${ }^{3}$, Thierry Leïchlé ${ }^{1}$ \\ Susan Trolier-McKinstry ${ }^{2}$ and Liviu Nicu ${ }^{1}$ \\ ${ }^{1}$ LAAS-CNRS, University of Toulouse, 7 Avenue du Colonel Roche, 31077 Toulouse, France \\ ${ }^{2}$ Department of Materials Science and Engineering and Materials Research Institute, The Pennsylvania State \\ University, N-227 Millennium Science Complex, University Park, PA 16802 USA \\ ${ }^{3}$ Laboratoire des Sciences de l'Information et des Systèmes, 8 boulevard Louis XIV 59000 Lille, France \\ E-mail: dsaya@laas.fr
}

\begin{abstract}
A piezoelectric nanoelectromechanical system (NEMS) with integrated actuation and detection capabilities was fabricated using lead zirconate titanate (PZT) thin films patterned by microcontact printing. PZT-coated cantilever resonators of various dimensions were fabricated to assess the variability in PZT properties as a function of the device dimensions; the microcontact-printed PZT was $281 \mathrm{~nm}$ thick. PZT layers of the cantilevers were poled at $107 \mathrm{kV} / \mathrm{cm}$ and $150{ }^{\circ} \mathrm{C}$ to improve their piezoelectric properties. It was demonstrated that PZT piezoelectrics can be utilized for simultaneous actuation and detection of resonance. The PZT cantilevers were analytically modelled to estimate values of their piezoelectric coefficient $d_{31}$. Mechanical tip displacement detection of the cantilevers by laser vibrometer was also carried out to validate the estimation of $d_{31}$.
\end{abstract}

\section{INTRODUCTION}

Over the last decade, advances in nanofabrication technology and electromechanical systems have led to the emergence of nanoelectromechanical systems (NEMS). NEMS-based resonators have significantly enhanced device performance through size reduction. ${ }^{1-12}$ Specifically, mechanical resonators with freestanding structures of nanometer scale cantilevers or bridges have high sensitivities for mass sensing applied to gas ${ }^{4,5}$ and biomolecule ${ }^{6,7}$ detection. NEMS structures are fabricated two ways; bottom-up fabrication produces nanoresonators with potentially smaller sizes and thus higher sensitivities in mass detection, ${ }^{4,5}$ topdown fabrication techniques enable mass production and integration with electrical circuits. ${ }^{6-12}$

Piezoelectric films, which translate mechanical energy into electrical and vice-versa, have widely been used for microelectromechanical systems (MEMS) transduction, i.e. actuation and detection. ${ }^{9-21}$ Of the various transduction mechanisms used in MEMS devices, piezoelectricity requires relatively low actuation voltage and offers the possibility of sensing, resulting in integrated devices that are compact, and thus potentially portable. ${ }^{13-16,20,21}$ In this regard, piezoelectric films could be particularly advantageous for transduction in NEMS, since there are few solutions suited to both actuation and sensing at this scale. However, studies have shown that some sputtered PZT films on NEMS resonators displayed reduced performance, ${ }^{17,18}$ likely as a result of a deterioration of the films' piezoelectric characteristics with reduced thickness. Recently, microcontact printed PZT films were found to retain their characteristics with reduced lateral dimensions and thicknesses. ${ }^{19,20}$

The aim of this work is thus to prove that microcontact printed PZT films are good candidates for efficient NEMS transduction. For this, silicon cantilevers of various dimensions integrating microcontact printed 
PZT thin film were fabricated and characterized. The PZT cantilevers were assessed for piezoelectric excitation and detection. The effect of poling on the piezoelectric characteristics of the PZT was investigated. Then, the PZT cantilevers were analytically modeled as laminated beams for estimations of their piezoelectric coefficient $d_{31}$, with a measure of the electrical charge created in the piezoelectric layer for a given excitation voltage around a given resonance. This method was also validated by resonance measurements by means of a laser vibrometer.

\section{FABRICATION OF SILICON CANTILEVERS WITH MICROCONTACT PRINTED PZT FILM}

Fabrication of PZT-coated cantilevers was carried out using SOI (silicon on insulator) wafers with a 340 $\mathrm{nm}$ top Si layer, $1 \mu \mathrm{m}$ thick buried $\mathrm{SiO}_{2}$ and a $525 \mu \mathrm{m}$ thick bulk Si handle wafer. An ultraviolet (UV) stepper photo repeater (I-line FPA 3000 Canon Inc.) was used for all lithography steps since high resolution and precise alignments were desirable for top-down NEMS fabrication.

First, a $100 \mathrm{~nm}$ thick $\mathrm{SiO}_{2}$ film was grown by thermal oxidation and a $60 \mathrm{~nm}$ thick hafnium dioxide $\left(\mathrm{HfO}_{2}\right)$ film was deposited by atomic layer deposition (ALD) to create a barrier against $\mathrm{Pb}$ diffusion (Fig. 1.1). The deposition occurred at $200{ }^{\circ} \mathrm{C}$ with a chamber pressure of 0.72 Torr. The reactant pulse time for $\mathrm{H}_{2} \mathrm{O}$ and tetrakis(ethylmethylamino)hafnium (TEMAH) was 0.03 and $0.15 \mathrm{~s}$ respectively, with a purge time of $10 \mathrm{~s}$ between each pulse. These cycles were repeated 619 times to create an amorphous $60 \mathrm{~nm}$ thick $\mathrm{HfO}_{2}$ layer which was crystallized with a $700{ }^{\circ} \mathrm{C}$ heat treatment in an $\mathrm{O}_{2}$ ambient for $1 \mathrm{~min}$. A $30 \mathrm{~nm}$ titanium adhesion layer was sputtered at room temperature using a power of $200 \mathrm{~W}$, a bias of $460 \mathrm{~V}$, a current of $0.47 \mathrm{~A}$, a deposition pressure of $2 \mathrm{mTorr}$, and an Ar flow rate of $8 \mathrm{sccm}$, for $300 \mathrm{~s}$. Without breaking vacuum, a 125 $\mathrm{nm}$ Pt bottom electrode was sputtered at room temperature using a power of $200 \mathrm{~W}$, a bias of $580 \mathrm{~V}$, a current of $0.34 \mathrm{~A}$, a deposition pressure of $2.5 \mathrm{mTorr}$, and an Ar flow rate of $11 \mathrm{sccm}$, for $417 \mathrm{~s}$ (Fig. 1.2). Next, the cantilever was defined by reactive ion etching (RIE). For this purpose, after patterning a photoresist layer with the stepper, a $540 \mathrm{~nm}$ nickel layer to be used as the hard mask during ion etching was deposited by electroplating. Successive reactive ion etching of $\mathrm{Pt}, \mathrm{Ti}, \mathrm{HfO}_{2}, \mathrm{SiO}_{2}$ and top $\mathrm{Si}$ layer was performed until reaching the buried $\mathrm{SiO}_{2}$ layer (Fig. 1.3). Then, the nickel hard mask was removed using the nickel etchant TFB (Nitric acid aqueous solution). Microcontact printing of the PZT film was then performed on top of the electrode patterned on the silicon structure (Fig. 1.4). For printing, a PZT sol-gel solution ( $1 \% \mathrm{Mn}$ doped, with atomic percentage $\mathrm{Pb}=110 \%, \mathrm{Zr}=30 \%, \mathrm{Ti}=70 \%, \mathrm{Mn}=1 \%$ ), from Mitsubishi Materials were spin-coated onto a polydimethylsiloxane (PDMS) stamp with a polyurethane layer that improved wetting of the PZT solution ${ }^{19}$. The PZT sol-gel solution was transferred onto the entire surface of the chip by manually pressing the stamp, and the film was pyrolyzed at $350{ }^{\circ} \mathrm{C}$ for $300 \mathrm{~s}$ and subsequently crystallized at $650{ }^{\circ} \mathrm{C}$ for $60 \mathrm{~s}$. This printing process was repeated seven times to achieve a final film thickness of $281 \mathrm{~nm}$ (Fig. 1.4). The resulting films showed microstructures characteristic of the perovskite phase, with a small amount of pyrochlore observed at triple points. Printed 30/70 PZT features exhibited a relative permittivity of 500 and a loss tangent of $0.9 \%$. The hysteresis loops were well formed, without pinching of the loops. The patterned features showed remanent polarizations of $27 \mu \mathrm{C} / \mathrm{cm}^{2}$, and coercive fields of $110 \mathrm{kV} / \mathrm{cm}$. Since the cantilever and bottom electrode was defined before the PZT printing step, as shown in Fig. 1.3, there was no need for precise alignment during stamping to achieve PZT film patterning on the cantilever.

In order to access the bottom electrode, the PZT film was patterned by wet etching in buffered hydrofluoric acid (BOE) after a photolithography step using SPR1813 photoresist (Fig. 1.5). Then, the Ti/Pt bottom 
electrode was patterned by reactive ion etching with a $6 \mu \mathrm{m}$ thick photoresist mask (Fig. 1.6) and a $30 \mathrm{~nm}$ thick Pt top electrode was patterned by lift off (Fig. 1.7). For this step, ZEP520A electron beam resist was patterned via electron beam lithography (EBPG 5200, Vistec) using a beam current of $40 \mathrm{nA}$, a dose of 365 $\mu \mathrm{C} / \mathrm{cm}^{2}$, an aperture size of $400 \mu \mathrm{m}$, and a beam step size of $25 \mathrm{~nm}$. After development of the photoresist, the Pt was sputtered and lifted off.

The next step was dedicated to patterning the ground plane, in order to allow the top Si layer of the SOI wafer to be electrically grounded (not shown in Fig. 1). This process consisted first of etching the $\mathrm{HfO}_{2}$ and $\mathrm{SiO}_{2}$ films to expose the top $\mathrm{Si}$ layer everywhere on the chip surface, except at the electrode and cantilever locations. Then Ti/Al $(100 \mathrm{~nm} / 700 \mathrm{~nm})$ was deposited on the exposed Si surface. This metallization of $\mathrm{Ti} / \mathrm{Al}$ was done at the same time as the metallization of the electrical circuits in the following step (Fig. 1.9). As discussed in section III. A, this step eliminated the parasitic capacitance between the electrodes and the device Si layer.

In order to prevent electrical shorts between the bottom electrode and the Ti/Al electrode deposited in the following process, a $100 \mathrm{~nm}$ thick $\mathrm{Si}_{3} \mathrm{~N}_{4}$ insulating film was patterned by lift-off using a bi-layer $300 \mathrm{~nm}$ thick LOR3A and $1 \mu \mathrm{m}$ thick ECI photoresist (Fig. 1.8). The LOR3A layer was used to facilitate the lift-off process due to the re-entrant profile. The $\mathrm{Si}_{3} \mathrm{~N}_{4}$ insulating film was deposited by inductively-coupled plasma chemical vapor deposition (ICPECVD) in order to ensure a uniform and conformal deposition over the step formed by the bottom electrode and PZT layer. Electrical connections were made by lift-off patterning of $100 \mathrm{~nm} / 700 \mathrm{~nm} \mathrm{Ti} / \mathrm{Al}$ using $2.6 \mu \mathrm{m}$ thick nLOF photoresist (Fig. 1.9). Finally, backside etching for cantilever release was performed by deep reactive ion etching of the handle Si wafer using a $40 \mu \mathrm{m}$ thick AZ40XT photoresist mask, followed by etching of the buried $\mathrm{SiO}_{2}$ layer (Fig. 1.10). Scanning electron microscopy photographs of the fabricated PZT covered cantilevers are shown in Fig. 2.
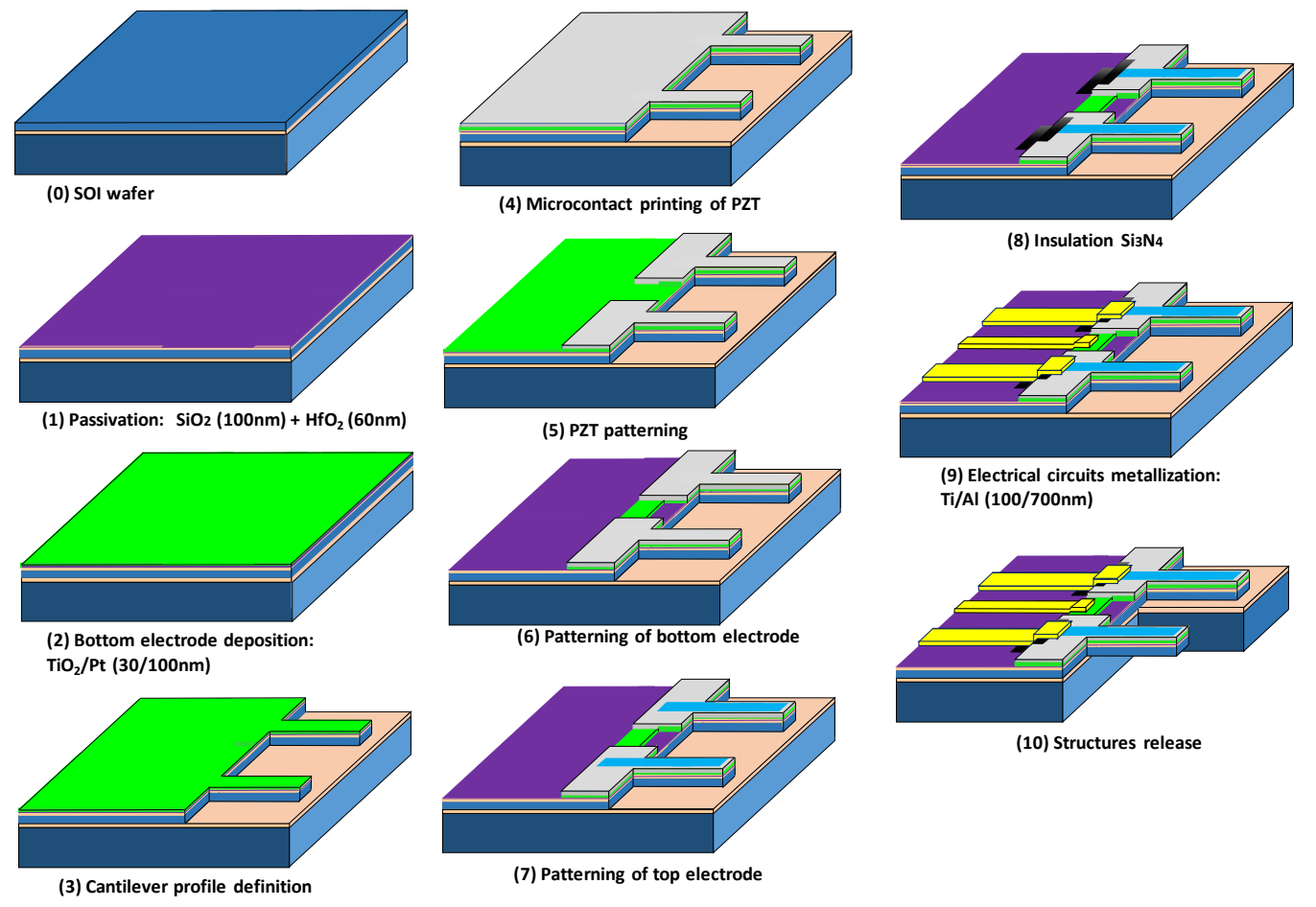

(8) Insulation $\mathrm{Si}_{3} \mathrm{~N} 4$

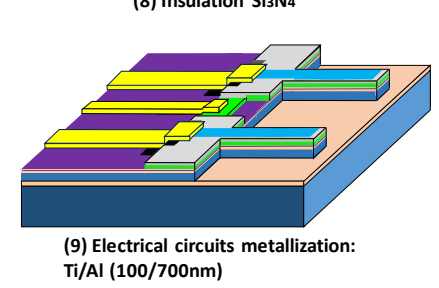
Ti/Al (100/700nm)

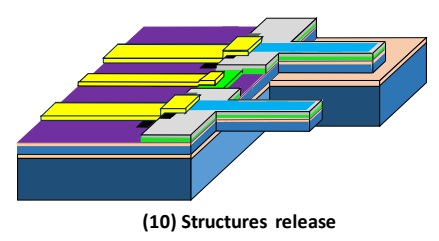

FIG. 1. Fabrication process of free-standing silicon cantilevers with microcontact printed PZT films. 


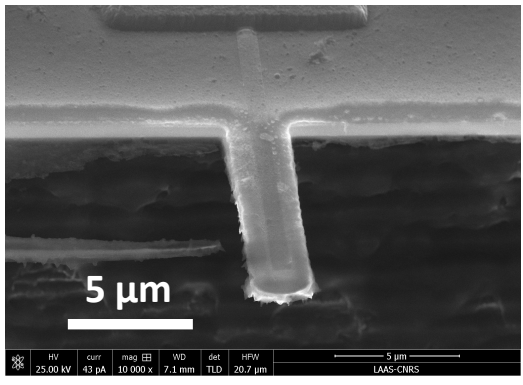

(a)

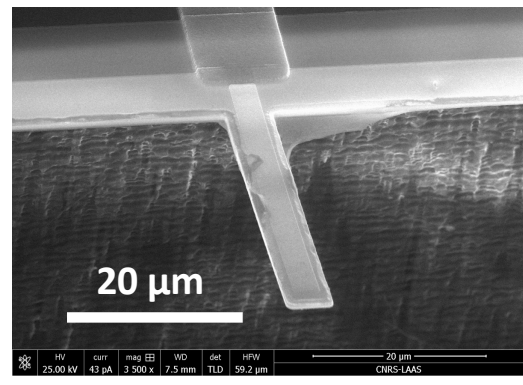

(b)

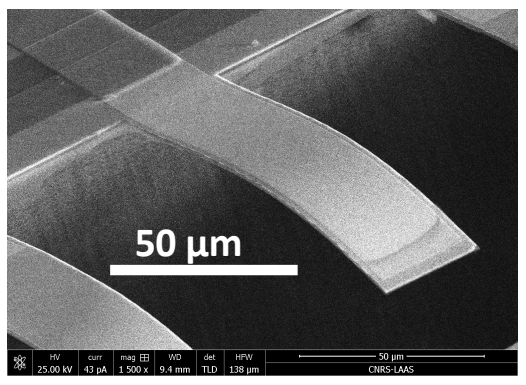

(c)

FIG. 2. SEM images of cantilevers with microcontact printed PZT films. (a) Short (10 $\mu \mathrm{m}$ long), (b) medium size (35 $\mu \mathrm{m}$ long) and (c) long (150 $\mu \mathrm{m}$ long) PZT coated cantilevers.

Each cantilever is a multilayer composed of $300 \mathrm{~nm}$ thick $\mathrm{Si}, 100 \mathrm{~nm}$ thick $\mathrm{SiO}_{2}, 60 \mathrm{~nm}$ thick $\mathrm{HfO}_{2}, 30$ $\mathrm{nm} / 100 \mathrm{~nm}$ thick Ti/Pt layers as the bottom electrode, $281 \mathrm{~nm}$ thick microcontact printed PZT layer, and 30 $\mathrm{nm}$ thick Pt top electrode, as shown in Fig. 3. An unreleased cantilever with exactly the same structure was integrated onto the same chip, serving as a reference to compensate the static capacitance of the piezoelectric stack during electrical characterization, as described in section III. A.

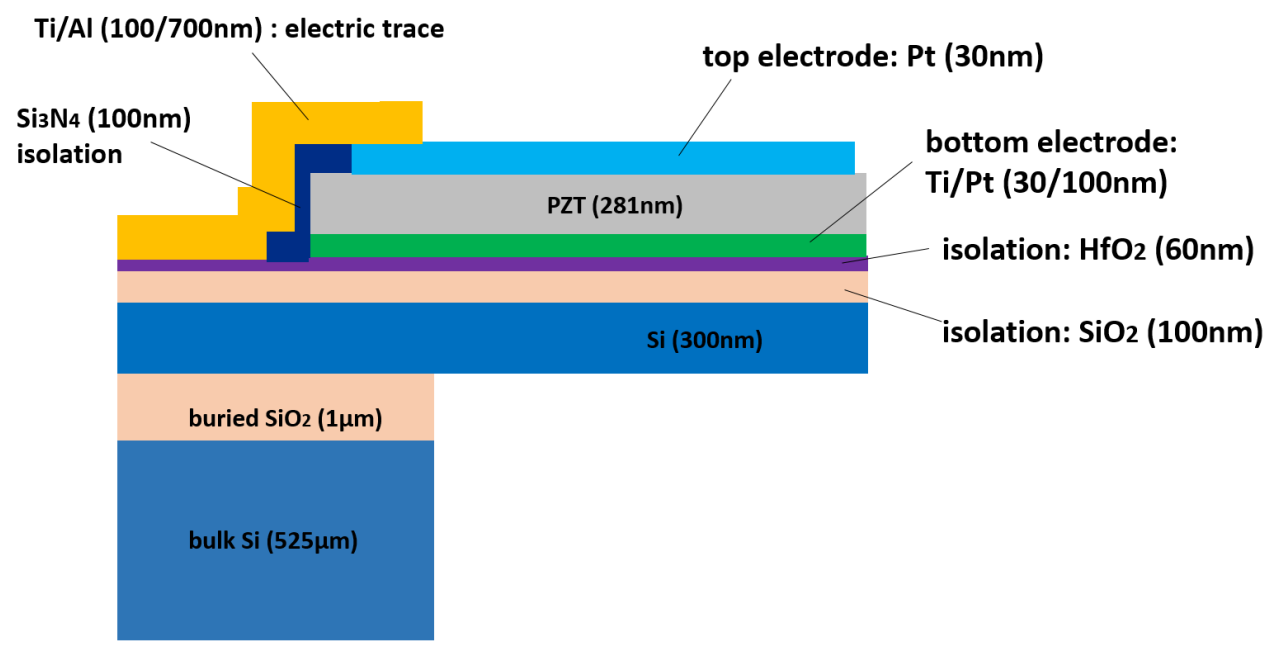

FIG. 3. Scheme of the integrated piezoelectric stack.

Three different types of cantilevers (depicted in Table I) were designed in order to study possible dimensional effects upon PZT film properties: the short cantilevers are $10 \mu \mathrm{m}$ long and $2 \mu \mathrm{m}$ wide. The medium size cantilevers are $35 \mu \mathrm{m}$ long, $5 \mu \mathrm{m}$ wide. Lastly, the long cantilevers are $150 \mu \mathrm{m}$ in length and $30 \mu \mathrm{m}$ in width.

TABLE I. Dimensions of the fabricated cantilevers.

\begin{tabular}{cccc}
\hline & Short cantilever & Medium cantilever & Long cantilever \\
\hline Length & $10 \mu \mathrm{m}$ & $35 \mu \mathrm{m}$ & $150 \mu \mathrm{m}$ \\
\hline $\begin{array}{c}\text { Width } \\
\text { (except top Pt layer) }\end{array}$ & $2 \mu \mathrm{m}$ & $5 \mu \mathrm{m}$ & $30 \mu \mathrm{m}$ \\
\hline Width (top Pt layer) & $1 \mu \mathrm{m}$ & $3 \mu \mathrm{m}$ & $26 \mu \mathrm{m}$ \\
\hline
\end{tabular}




\section{MEASUREMENT OF THE CANTILEVER RESONANCE FREQUENCY USING THE PZT PIEZOELECTRIC RESPONSE}

\section{A. Electrical set-up}

A schematic of the apparatus used for resonance frequency measurements ${ }^{16,20}$ is shown in Fig. 4. To operate the device, a sinusoidal voltage $\mathrm{V}_{1}$ was applied between the top and bottom electrodes on the piezoelectric layer, leading to the motion of free-standing cantilevers due to the converse piezoelectric effect. An opposite voltage $-\mathrm{V}_{2}$ was also applied to an unreleased structure with the same dimensions which served as a reference to reduce the effect of the static capacitance of the PZT layer. The charges generated by the two structures were collected by the common bottom electrode and were amplified through a charge amplifier ( $1 \mathrm{~V} / \mathrm{pC}$ gain). The signal was then injected into a demodulator to extract both amplitude and phase.

The top Si layer was grounded to eliminate the charges generated by the parasitic capacitances $C_{1}$ and $C_{2}$, and the charges from $\mathrm{C}_{\mathrm{s}}$ were compensated by the reference; where $\mathrm{C}_{1}$ is the parasitic capacitance between the top electrode trace line and the top Si layer of the SOI, $\mathrm{C}_{2}$ is the parasitic capacitance between the bottom electrode line and the top Si layer of the SOI, $\mathrm{C}_{\mathrm{s}}$ is the static capacitance of the PZT (dielectric) layer sandwiched between the top and bottom electrodes, and $\mathrm{C}_{\mathrm{mot}}$ is the motional capacitance corresponding to the charges generated by the PZT layer during the resonator motion due to the direct piezoelectric effect.
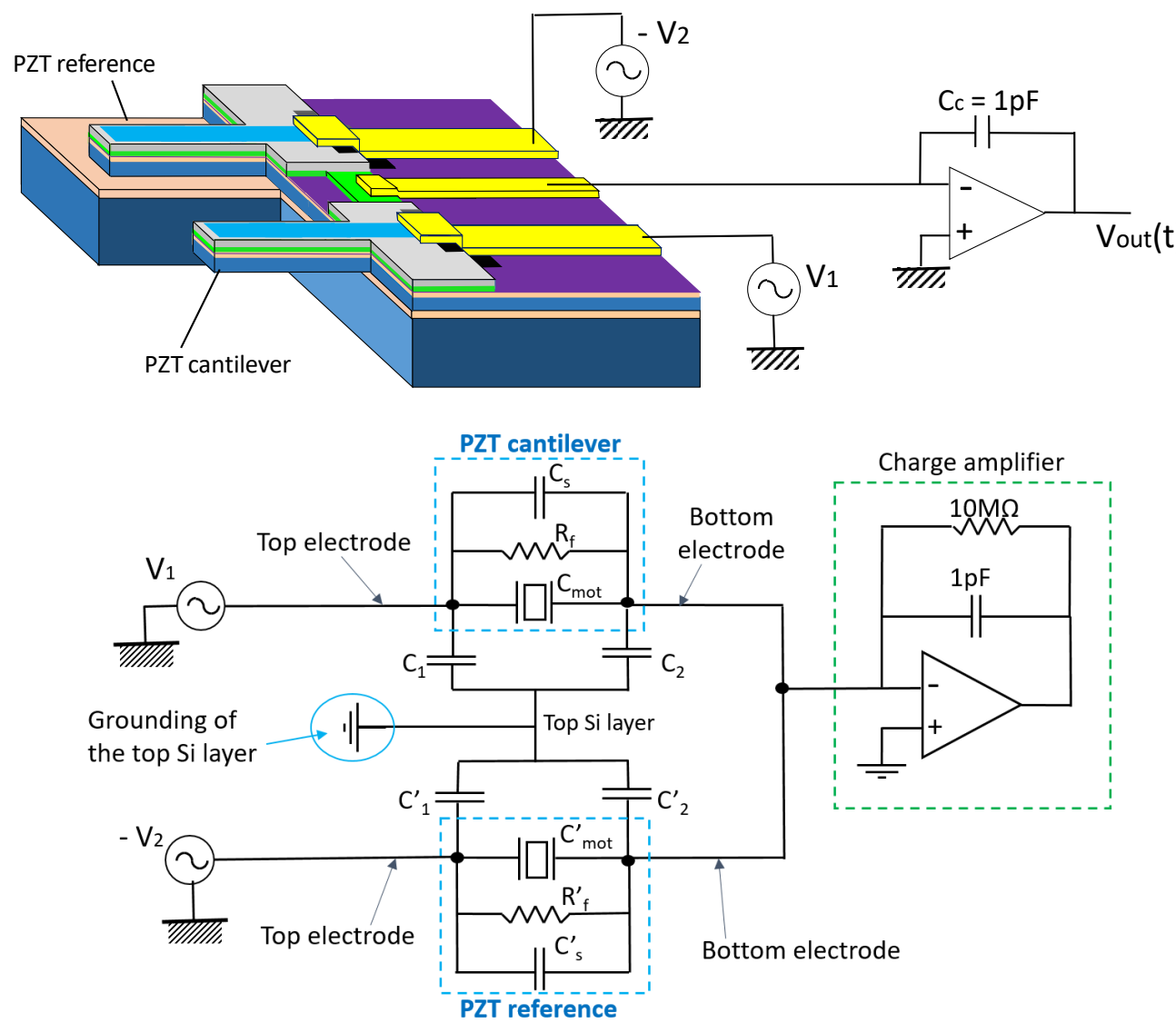

FIG. 4. Schematic sketch of electrical detection. Electrical connections of the device (up), details of the measurement electronics including the electrical model of the cantilevers (bottom). 


\section{B. Detection of resonance frequency by piezoelectric response and effect of poling on PZT films}

Using this electrical set up, the resonance frequencies of the medium size and long PZT cantilevers were detected through their piezoelectric responses. At first, the measurements were done without poling treatment of the PZT films. In order to improve the PZT layer properties and obtain a larger amount of charges upon cantilever deflection, the PZT was poled ${ }^{20,22-29}$ by applying $3 \mathrm{~V}$ between the top and bottom electrodes (i.e. $107 \mathrm{kV} / \mathrm{cm}$ across the $281 \mathrm{~nm}$ thick PZT layer) at a temperature of $150{ }^{\circ} \mathrm{C}$. A commercial Cascade Summit 12000 probe station allowed the Si chips to be heated while placing needle probes on the electrodes in order to pole the PZT and a Keithley 4200 was used to apply the DC voltage between the top and bottom electrodes.

The frequency spectra of the first mode resulting from the charges generated by the PZT layer with variation of poling time are shown in Fig. 5. In the case of the long cantilevers (Fig. 5(a)), even before poling, the resonance frequency could be detected using an actuation voltage $\left(\mathrm{V}_{1}\right)$ of $100 \mathrm{mV}$ as shown in Fig. 5(a) "Before poling". After 15 minutes of poling, the amplitude of the resonance peak increased by $33 \%$. By poling for $120 \mathrm{~min}$ more (for a total of $135 \mathrm{~min}$ ), the charges generated at the resonance peak increased further to $230 \mathrm{fC}$ (from an unpoled value of $120 \mathrm{fC}$ ). The detected resonance frequency of the first mode was $18.3 \mathrm{kHz}$ and quality factor in air was 67 . The second and third modes were also detected: $102.3 \mathrm{kHz}$ with a Q factor of 182 and $291.6 \mathrm{kHz}$ with a Q factor of 266, respectively.

In the case of the medium size cantilevers (Fig. 5(b)), the effect of poling was more remarkable. Before poling, the charge detected at resonance was only $1.4 \mathrm{fC}$ for an actuation voltage of $700 \mathrm{mV}$. However, after 15 minutes of poling, the charges generated at resonance were found to dramatically increase up to $10 \mathrm{fC}$ for an actuation voltage of $100 \mathrm{mV}$. By applying the poling protocol for $120 \mathrm{~min}$ more (for a total of 135 $\mathrm{min}$ ), the generated charges further increased to $29.6 \mathrm{fC}$. The detected resonance frequency of the first mode was $473 \mathrm{kHz}$ and the quality factor in air was 223. The second mode was $2.36 \mathrm{MHz}$ with Q factor 388 . For both long and medium size cantilevers, extending the poling treatment to a total of $255 \mathrm{~min}$ (120 min more) did not lead to any observable enhancement of the generated charges level (data not shown). Thus, 135 min of poling time maximized the PZT properties.

As for the short cantilever ( $10 \mu \mathrm{m}$ in length, $2 \mu \mathrm{m}$ in width), Figure 5(c) shows that before poling, the resonance frequency could not be detected. However, after performing a $135 \mathrm{~min}$ poling step, the resonance frequency was detectable. The amount of charges generated at resonance was $0.56 \mathrm{fC}$ for an actuation voltage of $20 \mathrm{mV}$. The measured resonance frequency was $5.72 \mathrm{MHz}$ with a quality factor in air of 381 . Hence, the capability of simultaneous actuation and detection of the cantilevers using microcontact printed PZT layers was validated even with the short cantilever.

For the medium and short cantilevers, the measured first mode resonance frequencies of $473 \mathrm{kHz}$ and 5.72 $\mathrm{MHz}$, respectively, are about $8 \%$ smaller than the simulated value of $508.0 \mathrm{kHz}$ and $6.215 \mathrm{MHz}$, obtained with the analytical model of a laminated beam described in section IV. A. ${ }^{30,31}$ As will be discussed in sections IV. A and IV. E, these slight differences may be attributed to non-ideal clamping of the cantilevers.

In the case of the long cantilever, the measured fundamental mode was $18.2 \mathrm{kHz}$ while the simulated one was $27.75 \mathrm{kHz}$. Hence, the difference between experimental and theoretical resonance frequency values reaches $52 \%$ in the case of long cantilevers. This large discrepancy is believed to be a consequence of a decrease in the cantilever's thickness due to overetching during fabrication. According to the analytical model which will be mentioned in section IV. A, the Si layer thickness of the long cantilever may be 
decreased to $90 \mathrm{~nm}$. It is because of non-uniformity in etching speed of the deep reactive ion etching of the handle Si wafer during the release of the cantilever structures (Fig. 1.10). The etching speed is slower on the edge of the etching patterns. The medium and the short cantilevers are situated on the edge of the etching patterns. Hence, while the etching for releasing long cantilever is about to be finished, the medium and the short cantilevers are not yet released. To complete the release process of all cantilevers, the long cantilevers may suffer from this overetching.

As can be seen in Fig. 5, the measured resonance frequencies slightly increase after the 135 min poling treatment, for the long cantilever from $18.2 \mathrm{kHz}$ (before poling) to $18.3 \mathrm{kHz}$ (after $135 \mathrm{~min}$ poling) and for the medium cantilever from $466.6 \mathrm{kHz}$ (before poling) to $473.2 \mathrm{kHz}$ (after $135 \mathrm{~min}$ poling). This results can be attributed to the remanent strain induced by the alignment of the PZT dipoles towards the direction of the applied field during poling, ${ }^{24,25}$ the resulting in-plane contraction ${ }^{26,27}$ should have produced a curvature change of the cantilevers. For these poling conditions, aging ${ }^{28,29}$ was limited, as demonstrated by the stability of the resonance peak amplitudes during measurements spanning a period of two months.

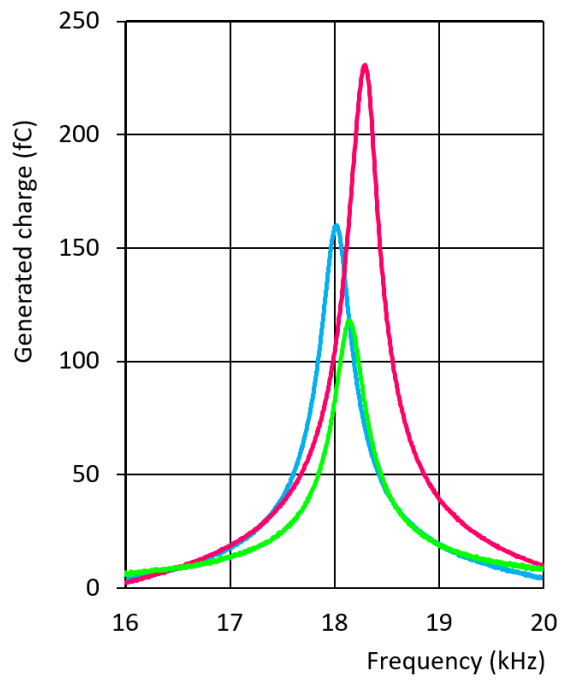

(a) Long cantilever

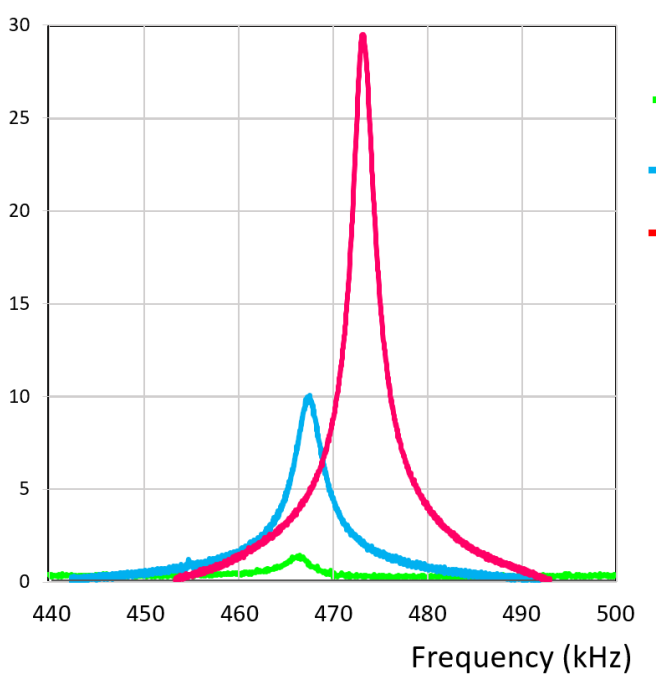

(b) Medium cantilever

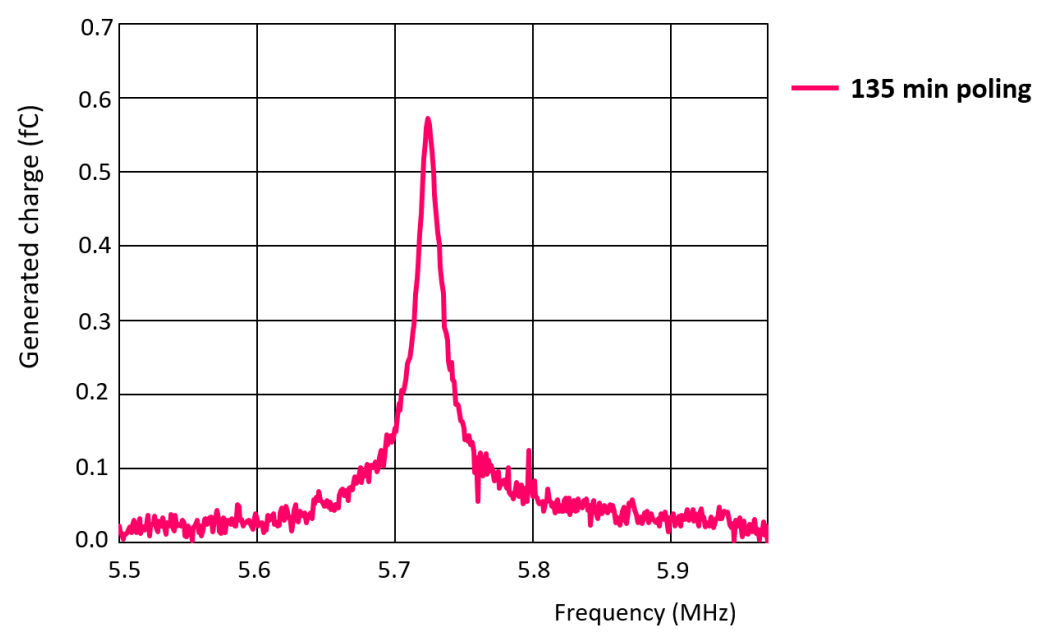

(c) Short cantilever 
FIG. 5. Resonance spectra with variation of poling time. (a) $150 \mu \mathrm{m}$ long cantilever actuated with $\mathrm{V}_{1}=$ $100 \mathrm{mV}$, (b) $35 \mu \mathrm{m}$ long (medium size) cantilever actuated with $\mathrm{V}_{1}=700 \mathrm{mV}$ before poling and $100 \mathrm{mV}$ after poling, and (c) $10 \mu \mathrm{m}$ long (short size) cantilever actuated with $\mathrm{V}_{1}=20 \mathrm{mV}$ after poling.

\section{ESTIMATION OF THE PIEZOELECTRIC COEFFICIENT $\boldsymbol{d}_{31}$}

Estimation of the piezoelectric coefficient $d_{31}$ was done by modeling the PZT cantilever as a laminated beam and by comparing the result of the model to two sets of experimental results at a particular resonance. First, the motional capacitance $C_{\text {mot }}$ was obtained from impedance data at resonance, from which the piezoelectric response was extracted. Secondly, the displacement of the tip of the cantilever as a function of the voltage applied to the piezoelectric layer was detected by a laser vibrometer. This was modeled using equivalent single layer beam theory for the laminated beam, with all details given in the Appendix. The following section presents a comparison of the analytical model and finite element model (FEM, ABAQUS) used for model validation and then the method used to determine the $d_{31}$ coefficient.

\section{A. Validation of the beam model with finite element computations - modal analysis}

As explained in the Appendix, the $k^{\text {th }}$ natural frequency is given by the equivalent single layer beam model of the cantilever: ${ }^{30}$

$$
f_{k}=\frac{\beta_{k}^{2}}{2 \pi L} \sqrt{\frac{D}{m}}
$$

with $\beta_{1}=1.875, \beta_{2}=4.694$, etc. and where $D$ is the bending stiffness and $m$ is the mass per unit length of the beam. These results were compared with a three-dimensional finite element model of the structures, built in the commercial code ABAQUS. The three cantilevers defined in Tables I and II were considered. ${ }^{32,33}$ The finite-element meshes are shown in Fig. 6. Each physical layer of the cantilevers is meshed with one layer of hexahedral finite elements with 20 nodes and quadratic shape functions (C3D20 finite elements), that include electric degrees of freedom for the PZT layer (C3D20E finite elements). The convergence was checked with refined meshes; those results are less than $0.1 \%$ different than the meshes of Fig. 6 . Figure 7 shows the mode shapes of some bending modes of the cantilevers obtained by finite element simulations with ABAQUS.

TABLE II. Properties of layers of the cantilever. ${ }^{32,33}$

\begin{tabular}{lccc}
\hline & $\begin{array}{c}\text { Thickness } \\
(\mathrm{nm})\end{array}$ & $\begin{array}{c}\text { Mass density } \\
\left(\mathrm{kg} / \mathrm{m}^{3}\right)\end{array}$ & $\begin{array}{c}\text { Young's } \\
\text { modulus (GPa) }\end{array}$ \\
\hline $\mathrm{Pt}$ (top) & 30 & 21450 & 145 \\
\hline $\mathrm{PZT}$ & 281 & 7800 & 105 \\
\hline $\mathrm{Pt}$ (bottom) & 100 & 21450 & 145 \\
\hline $\mathrm{Ti}$ & 30 & 4510 & 110 \\
\hline $\mathrm{HfO}_{2}$ & 60 & 9700 & 57 \\
\hline $\mathrm{SiO}_{2}$ & 100 & 2150 & 70 \\
\hline $\mathrm{Si}$ & 300 & 2500 & 169 \\
\hline
\end{tabular}




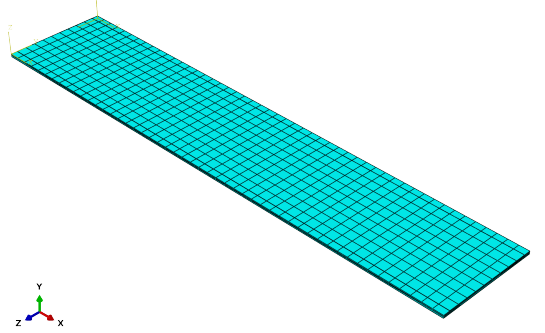

150 um long cantilever

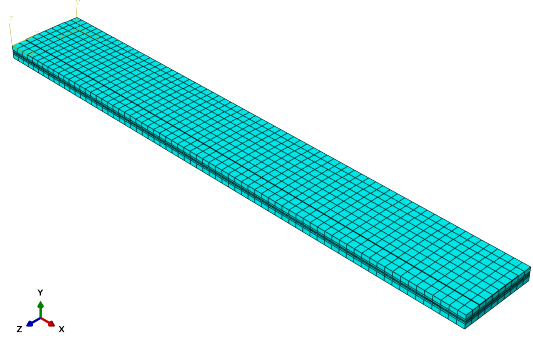

35 um medium cantilever

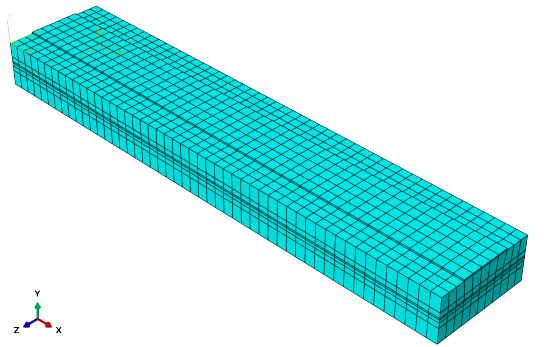

$10 \mu \mathrm{m}$ short cantilever

FIG. 6. Meshes used in the FE computations.

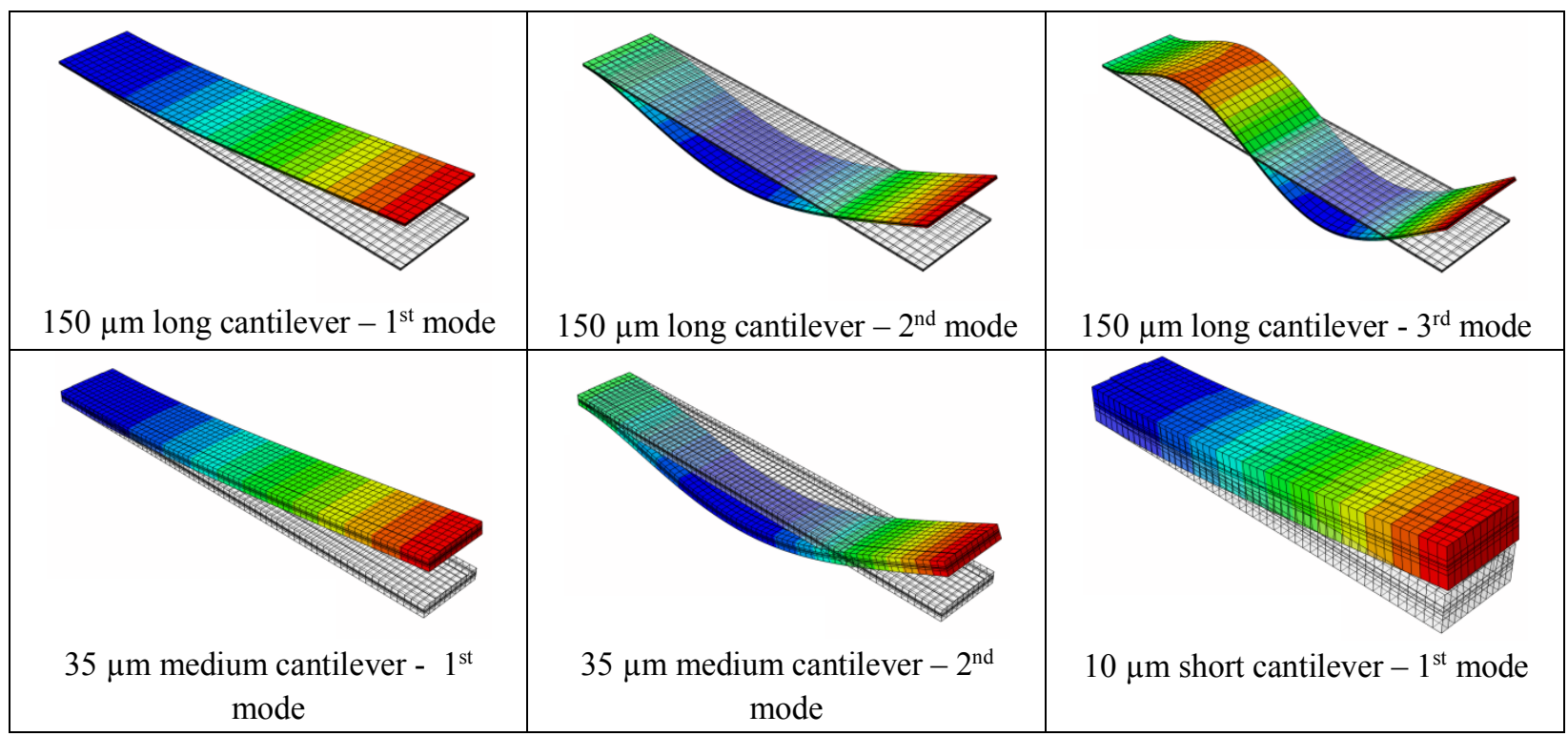

FIG. 7. Mode shapes of some bending modes of the cantilevers obtained by finite element simulations with ABAQUS.

Figure 8 and Table III show the natural frequencies of the beam obtained by the single layer model as a function of the ones obtained by the finite element computations. A difference of about $3 \%$ is observed between the two approaches. Considering the aspect ratios of the cantilevers (close to a thin plate for the $150 \mu \mathrm{m}$ long cantilevers and close to a thick beam with a small aspect ratio for the $10 \mu \mathrm{m}$ short cantilevers), as well as their complex lamination (7 layers), this error can be considered very small, which validates the equivalent single layer beam model. We also compared the natural frequencies of the cantilevers for different electrical boundary conditions (without piezoelectric effects, PZT layer in short circuit and PZT layer in open circuit) with $d_{31}=10 \mathrm{pm} / \mathrm{V}$ for the piezoelectric coefficient. Differences less than $0.1 \%$ where noticed, which is consistent with the small piezoelectric coupling factor (less than $1 \%$ ) associated to this $d_{31}$ value (see a reference ${ }^{34}$ for details about the piezoelectric effect on the natural frequencies in short and open circuit and their relation to the electromechanical coupling factor).

The experimental frequencies of the cantilevers of section III. B are also shown in Fig. 8. A line of slope 1 is provided, showing good agreement between the experimental and theoretical values of the resonance frequencies. It is notable that the experimental natural frequencies are smaller than those predicted by the theory. This can probably be explained by the non-ideal clamping with a smaller stiffness in experiments 
than in theory, as will be shown in Fig. 14, as well as some unavoidable uncertainties on the material constants values of the six materials used in the complex lamination of the beams. In the case of the long cantilever, the reduction in experimental natural frequency was caused by overetching during fabrication, as discussed in section III. B.

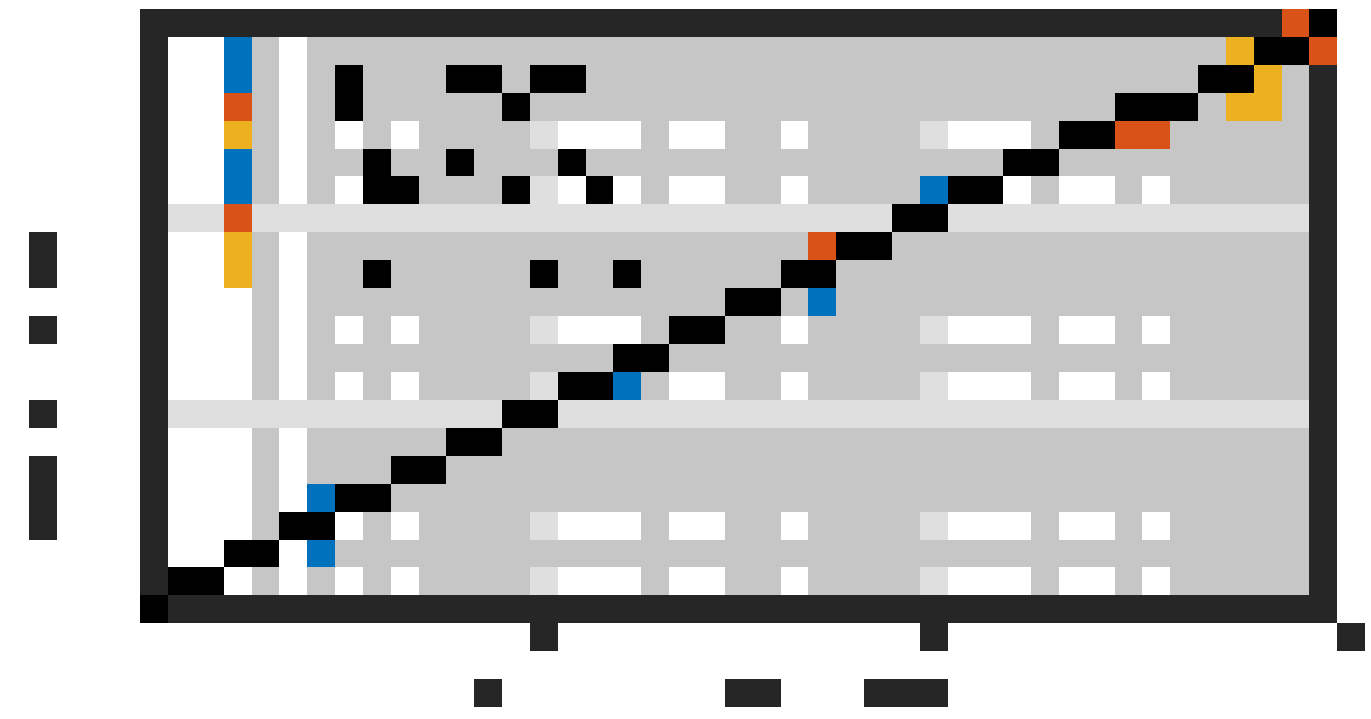

FIG. 8. Experimental (Exp.) resonance frequencies and finite element (ABAQUS) natural frequencies as a function of theoretical (equivalent single layer beam theory) natural frequencies of the cantilevers. The dashed line has a slope of one.

TABLE III. Comparison of natural frequencies from the equivalent single layer beam theory and the reference 3D finite element computation.

\begin{tabular}{|c|c|c|c|c|c|c|}
\hline & \multicolumn{3}{|c|}{$\begin{array}{c}150 \mu \mathrm{m} \text { long } \\
\text { cantilever }\end{array}$} & \multicolumn{2}{c|}{$\begin{array}{c}35 \mu \mathrm{m} \text { medium } \\
\text { cantilever }\end{array}$} & $\begin{array}{c}10 \mu \mathrm{m} \text { short } \\
\text { cantilever }\end{array}$ \\
\hline & Mode 1 & Mode 2 & Mode 3 & Mode 1 & Mode 2 & Mode 1 \\
\hline $\begin{array}{c}\text { Single layer beam } \\
\text { theory [kHz] }\end{array}$ & 27.75 & 173.95 & 487.06 & 508.03 & 3184.1 & 6214.5 \\
\hline $\begin{array}{c}\text { Finite elements } \\
{[\mathrm{kHz}]}\end{array}$ & 27.09 & 169.54 & 475.44 & 492.63 & 3074.8 & 5985.90 \\
\hline Error [\%] & 2.39 & 2.53 & 2.39 & 3.0 & 3.4 & 3.68 \\
\hline
\end{tabular}

\section{B. Validation of the beam model with finite element computations - frequency response functions with piezoelectric effect.}

The mechanical frequency response (FRF) of the cantilevers determined by the equivalent single layer theory and by realistic finite-element computations was compared. A sinusoidal voltage of frequency $\Omega$ was applied to the PZT layer $V(t)=V_{0} \cos \Omega t$. The resulting tip displacement $w_{t}(t)=w_{t 0} \cos (\Omega t+\varphi)$ of the cantilevers by both models was then calculated and compared. The amplitude of the frequency response is simply $H(\Omega)=w_{t} / V_{0}$. For the finite-element computations, the frequency response is obtained at each frequency $\Omega$ with an inversion of the dynamical stiffness matrix (direct steady-state dynamics in ABAQUS). A mass-proportional damping is used, for which the damping matrix is $C=a M$, where $M$ is the mass matrix. 
$a$ was chosen so that quality factors between 10 to 200 were obtained for the modes of interest for the three cantilevers (short cantilever: $a=10^{6} \mathrm{~s}^{-1}$, medium cantilever: $a=50.10^{3} \mathrm{~s}^{-1}$, long cantilever: $a=$ $\left.20.10^{3} \mathrm{~s}^{-1}\right)$. For the $k^{\text {th }}$ angular natural frequency, $\omega_{k}$, computed by the finite element model, $Q_{k}=\omega_{k} / \alpha$. For the analytical model, Eq. (A.11) and (A.14) in the Appendix are used. All the other parameters $\left(\chi_{k}, m\right.$, $L$ ) were computed with the geometrical and material characteristics of the cantilevers (Tables I and II). Moreover, the width $b_{p}$ of the piezoelectric layer used in Eq. (A.4) in the Appendix to compute the piezoelectric constants $\Theta$ and $\Xi$ was set equal to the top electrode width (see Table I), since most of the electric field lines created in the PZT layer encompass the volume just below the top electrode. Besides, in the analytical model, the electric field in the piezoelectric layer is independent of $y$.

Figure 9 shows $H(\Omega)$ for the three cantilevers, computed by the single layer theory and the finite-element model. Very good agreement is obtained for the three cantilevers, with a slight underestimation of the frequency response by the analytical model. This effect is mainly observed in the case of the first mode of the short cantilever. This can be explained by the relatively large aspect ratio of this cantilever (the ratio between its length and the cross-sectional thickness is 11 for the short cantilever, see Fig. 10), for which the Euler-Bernoulli theory is at its limits. Moreover, as shown in Fig. 10, there are fringing electric field lines in the PZT layer that extend beyond the top electrode, which results in an increased active PZT volume with respect to that considered in the analytical model. Those effects are smaller for the medium and the long cantilever, with larger aspect ratios ( 38 and 166, respectively). Given the good agreement between measured and modeled response, the analytical model can be used for the $d_{31}$ piezoelectric coefficient estimations.
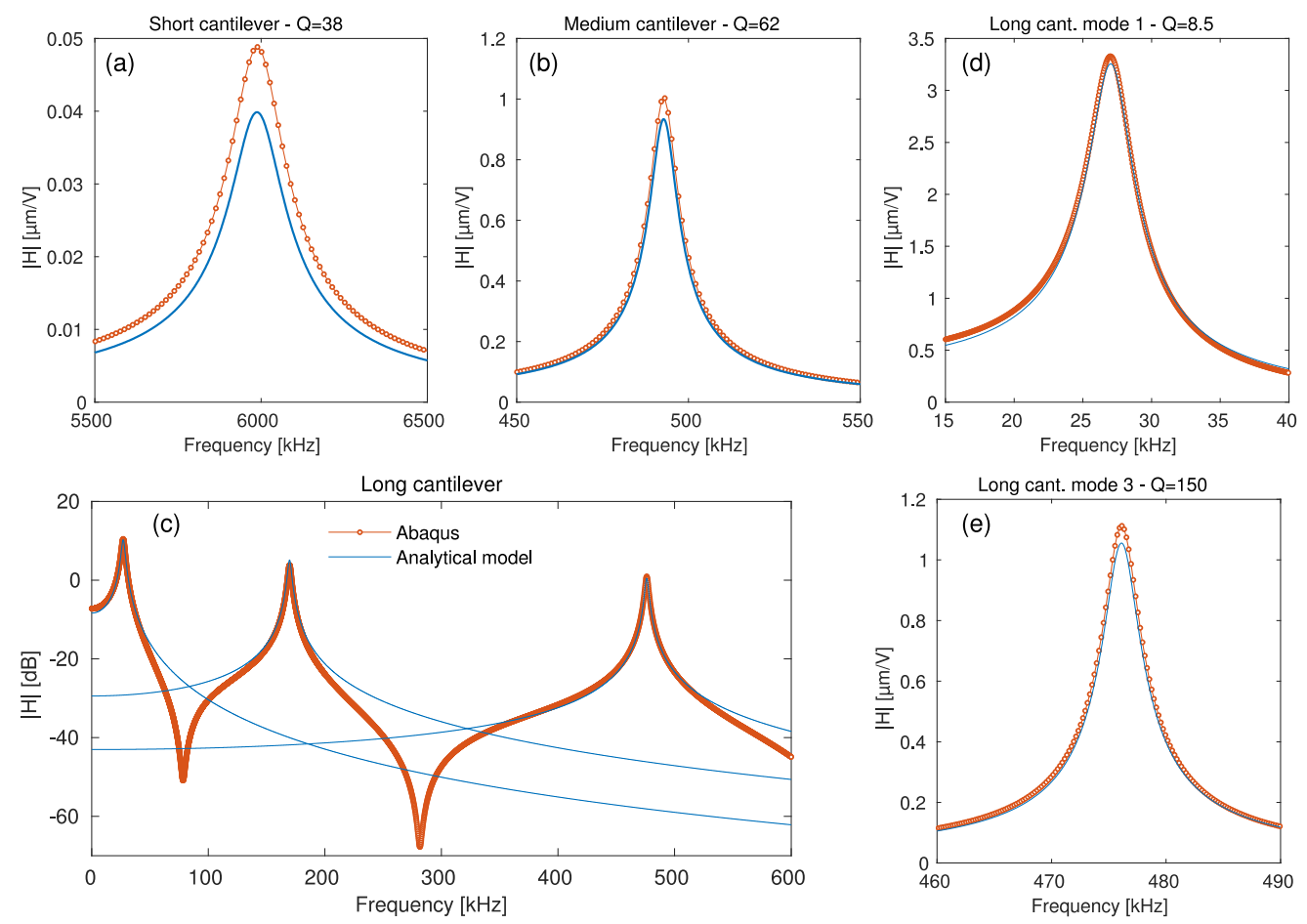

FIG. 9. Frequency response functions: tip displacement of the cantilevers for a unit voltage in the PZT layer as a function of driving frequency. Comparison between reference finite element computation results with ABAQUS (orange lines) and results from the equivalent single layer model (blue lines. Solid: 4 mode model; dashed: single mode model). 


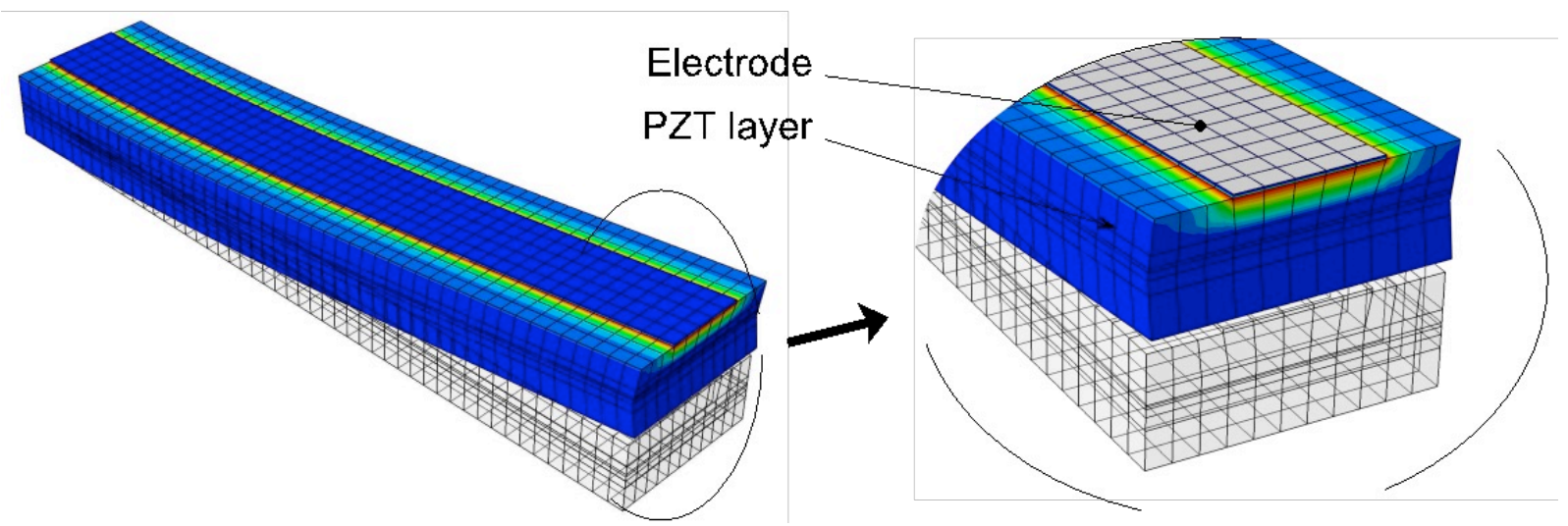

FIG. 10. Deformed shape at the first resonance of the short cantilever with the electrical potential shown in color.

\section{Estimation of the $d_{31}$ coefficient}

The $d_{31}$ piezoelectric coefficient was estimated by measuring the motional capacitance $C_{\text {mot }}$ of the cantilevers (the ratio between the number of piezoelectrically created electric charges and the input voltage) at a specific resonance. The piezoelectric response in the analytical model was then adjusted so that the modeled response fit the experimental data. This method has been used successfully in a previous work. ${ }^{18}$ As shown in the Appendix (Eq. (A.19), the motional capacitance $C_{\text {mot }}$ for the cantilevers can be written as:

$$
C_{\mathrm{mot}}=\alpha d_{31}^{2} \quad \text { with } \quad \alpha=\frac{Q_{k} Y_{p}^{2} \widehat{\widehat{\Theta}}^{2} \Phi_{k}^{\prime 2}(1)}{m L^{3} \omega_{k}^{2}}
$$

The $\alpha$ parameter $([\mathrm{N} / \mathrm{m}])$ is a function of the $k^{\text {th }}$ eigenmode (quality factor $Q_{k}$, natural frequency $\omega_{k}$ and mode shape slope at the tip $\Phi_{k}^{\prime}$ ), the Young's modulus of the PZT layer $Y_{p}$, the length $L$ of the cantilever and the piezoelectric constant $\hat{\Theta}$ proportional to the PZT layer mean height with respect to the neutral line of the cantilever.

For each cantilever, $d_{31}$ is estimated by:

$$
d_{31}=\sqrt{\frac{C_{\mathrm{mot}}}{\alpha}}
$$

where $C_{\text {mot }}$ is obtained by recording the resonance spectra as a function of actuation voltage and $\alpha$ is computed using the values of Tables I and II for $Y_{p}, L$ and $\hat{\Theta}$, the theoretical mode shapes for $\Phi_{k}^{\prime}$ (Eq. (A.10) in the Appendix) and the experimental values of $Q_{k}$ and $\omega_{k}$.

Figure 11(a) shows the $1^{\text {st }}$ mode resonance response of the motional capacitance (for cantilevers after 135 min poling treatment). A plot of the generated charges as a function of actuation voltages at resonance shows excellent linear behavior (Fig. 11(b)), permitting determination of the experimental values of $C_{\text {mot }}$. In the case of the long PZT cantilever, the applied voltage $\mathrm{V}_{0}$ varied from $25 \mathrm{mV}$ to $125 \mathrm{mV}$ (Fig. 11(1)) and in the case of the medium PZT cantilever, it varied from $50 \mathrm{mV}$ to $300 \mathrm{mV}$ (Fig. 11(2)). The average quality factor and experimental values of $C_{\text {mot }}$ were estimated to be 67 and 223, and $2.306 \mathrm{fC} / \mathrm{mV}$ and $0.296 \mathrm{fC} / \mathrm{mV}$, respectively for the long and medium cantilever. For the short PZT cantilevers, with applied voltage $\mathrm{V}_{0}$ 
ranging from $10 \mathrm{mV}$ to $100 \mathrm{mV}$ (Fig. 11(3)), the average quality factor and the experimental values of $C_{\text {mot }}$ were estimated to be 381 and $0.026 \mathrm{fC} / \mathrm{mV}$.

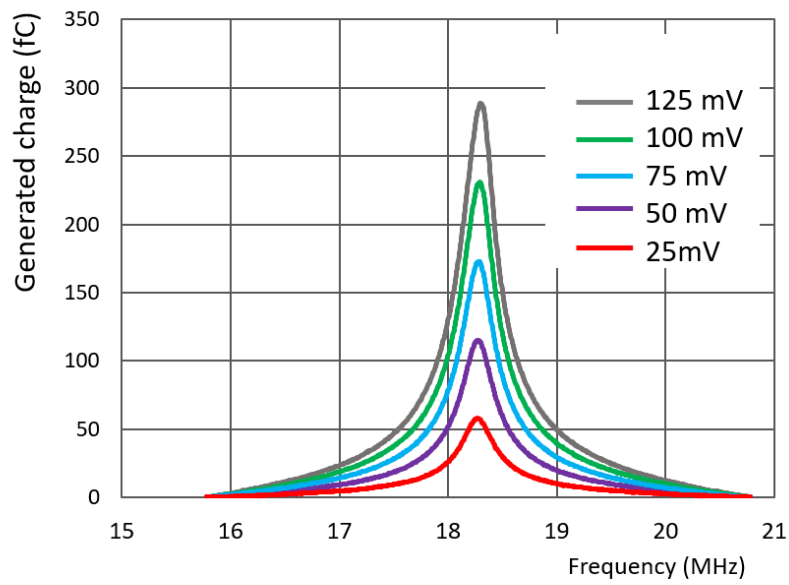

(a)

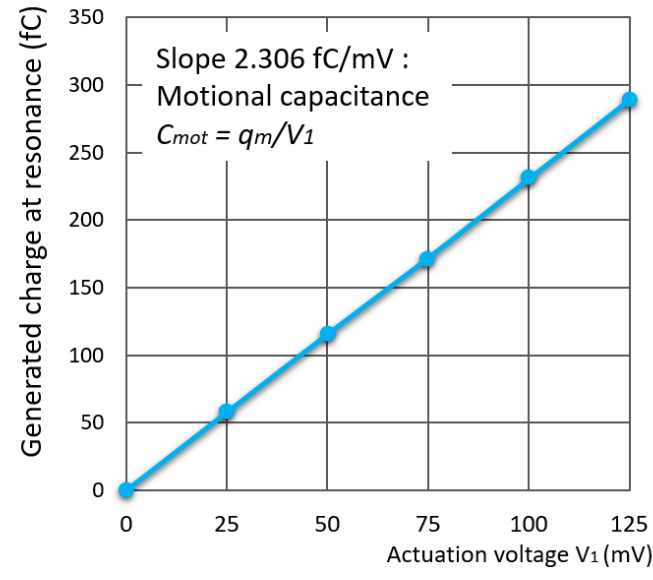

(b)

(1) Long PZT cantilever: $150 \mu \mathrm{m}$ in length, $30 \mu \mathrm{m}$ in width

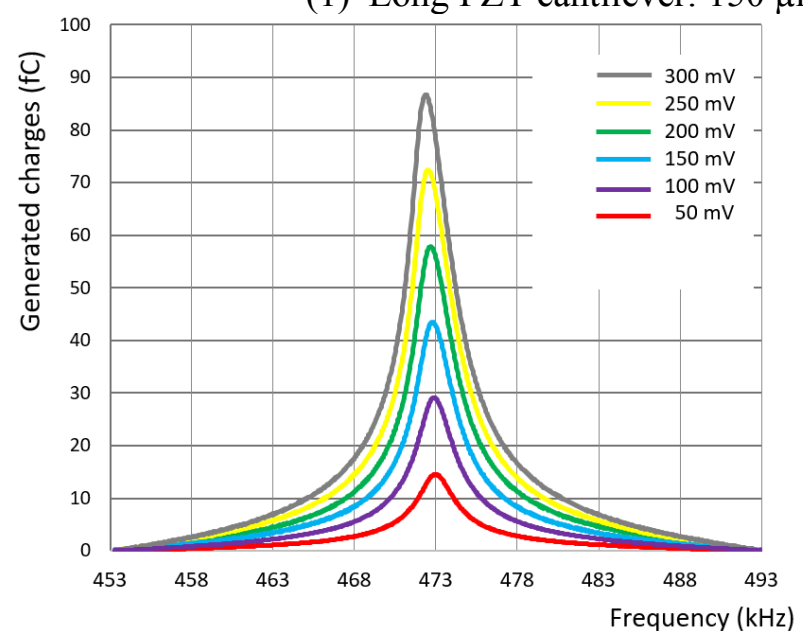

(a)

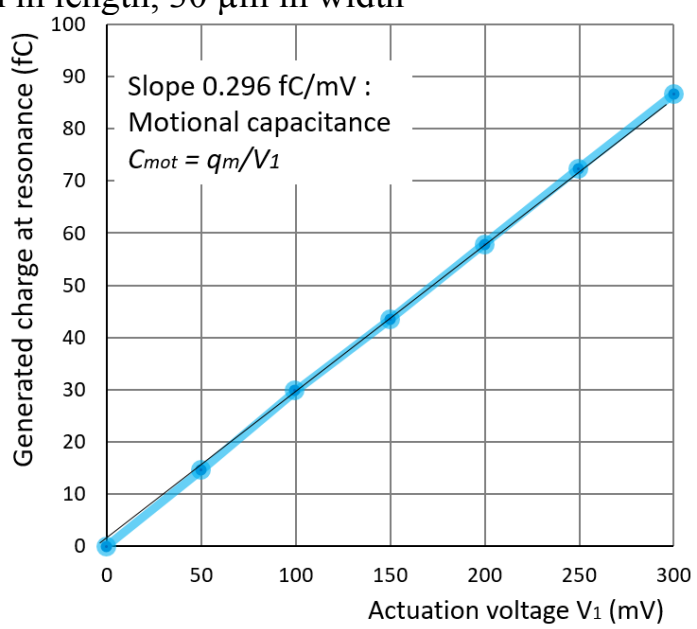

(b)

(2) Medium PZT cantilever: $35 \mu \mathrm{m}$ in length, $5 \mu \mathrm{m}$ in width

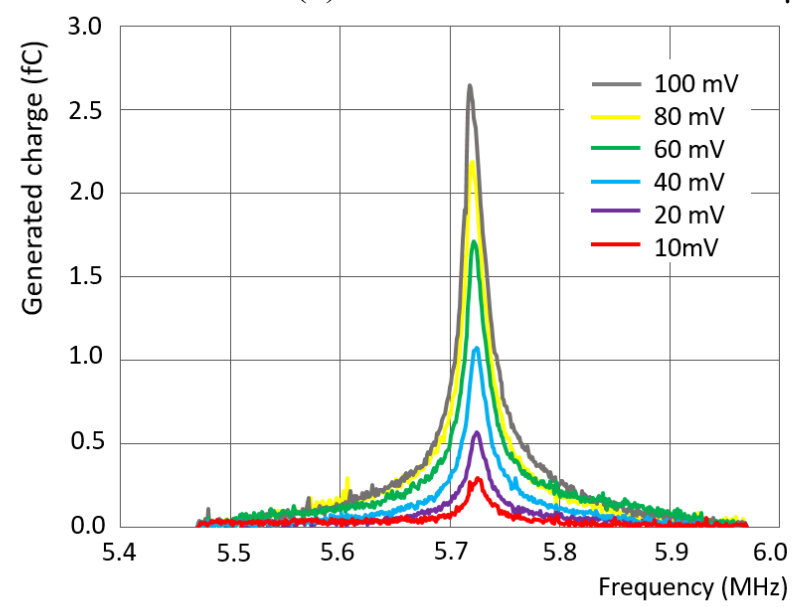

(a)

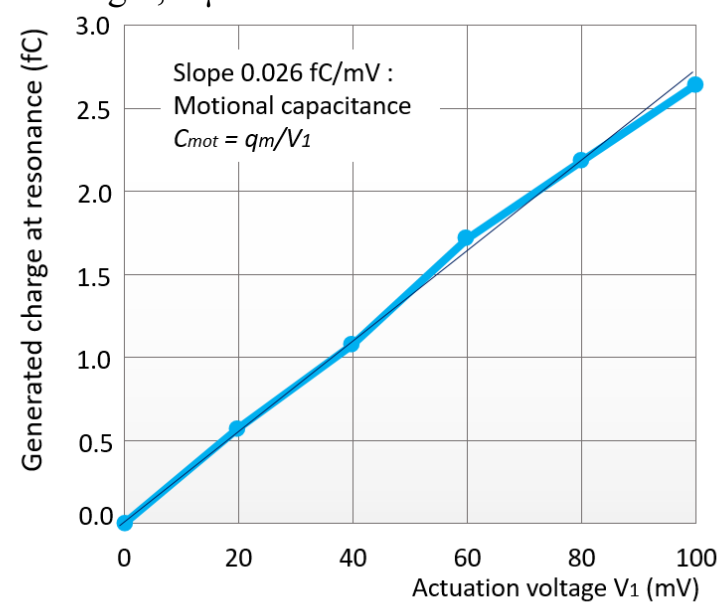

(b)

(3) Short PZT cantilever: $10 \mu \mathrm{m}$ in length, $2 \mu \mathrm{m}$ in width 
FIG. 11. (a) Piezoelectric responses of the PZT cantilevers as a function of actuation voltage after a 135 min poling process, (b) slope of the generated charge at the resonance peak as a function of actuation voltage.

\section{Estimated values of the $d_{31}$ coefficient and its evolutions with poling time}

The piezoelectric coefficient $d_{31}$ was calculated using the motional capacitance $C_{\text {mot }}$ and quality factors estimated in the previous section. Piezoelectric coefficients $d_{31}$ of $-6.9 \pm 0.1 \mathrm{pm} / \mathrm{V},-12.0 \pm 2.0 \mathrm{pm} / \mathrm{V}$ and $-11.0 \pm 2.4 \mathrm{pm} / \mathrm{V}$ were calculated respectively for the long, medium size and short cantilevers for the first mode. These calculated values of $d_{31}$ were smaller than those previously reported, ${ }^{19,21}$ where values of $d_{31}$ were estimated to be around $50 \mathrm{pm} / \mathrm{V}$ for PZT films with a thickness in the $100-200 \mathrm{~nm}$ range. These lower values are due to the use of a PZT composition off of the morphotropic phase boundary, as well as potential degradation of PZT layer induced by the presence of hydrogen ${ }^{35}$ most probably during ICPECVD process for deposition of $\mathrm{Si}_{3} \mathrm{~N}_{4}$ film (Fig. 1.8), even though annealing of $400{ }^{\circ} \mathrm{C} 30 \mathrm{~min}$ in $\mathrm{N}_{2}$ atmosphere was conducted to eliminate hydrogen molecules from the PZT layer.

The $d_{31}$ values were calculated for each poling time for serval cantilevers of each size. The evolution of $d_{31}$ values as a function of poling time are shown in Fig. 12 and Table IV. In the case of the medium size cantilever, the generated charges at resonance before poling treatment were very low (1.4 fC with $700 \mathrm{mV}$ actuation voltage), leading to $1.2 \mathrm{pm} / \mathrm{V}$ for the $d_{31}$ coefficient which is not unexpected for an unpoled piezoelectric. However, after 135 minutes of poling, the $d_{31}$ value became comparable to that of the PZT film integrated into the long cantilever. As for the short cantilever, the $d_{31}$ value was also comparable to that of the long cantilever PZT film after the poling process. The $d_{31}$ values did not show any significant increase when the poling time exceeded $135 \mathrm{~min}$.

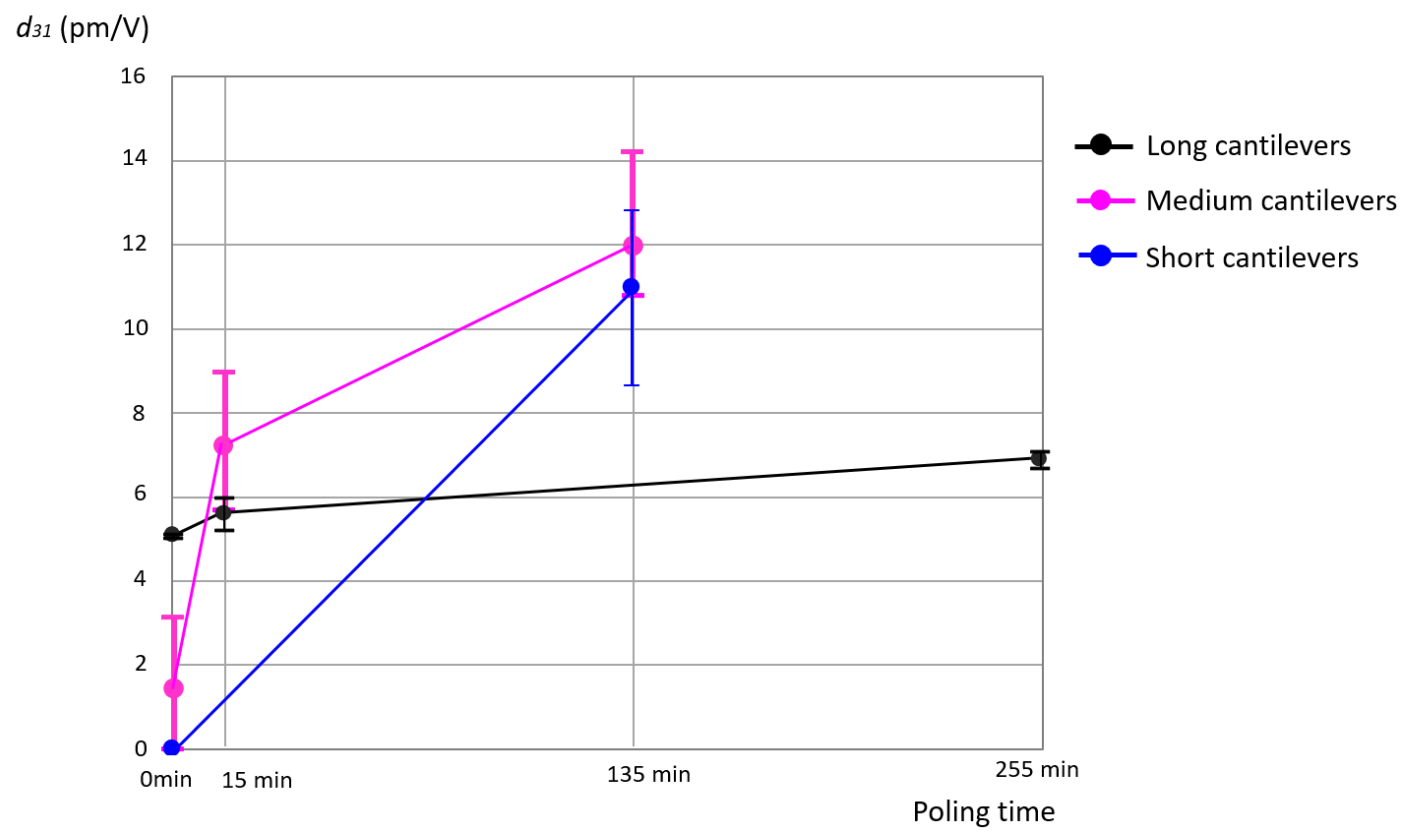

FIG. 12. Evolution of $d_{31}(\mathrm{pm} / \mathrm{V})$ as a function of poling time $\left(107 \mathrm{kV} / \mathrm{cm}\right.$ at $\left.150^{\circ} \mathrm{C}\right)$, for short, medium and long size cantilevers. 
TABLE IV. Evolution of $d_{31}(\mathrm{pm} / \mathrm{V})$ values, resonance frequencies and $\mathrm{Q}$ factor as a function of poling time.

\begin{tabular}{|c|c|c|c|c|c|}
\hline & Poling time & $\begin{array}{l}\text { Before } \\
\text { poling }\end{array}$ & $15 \mathrm{~min}$ & $135 \mathrm{~min}$ & $255 \mathrm{~min}$ \\
\hline \multirow{3}{*}{$\begin{array}{c}\text { Long } \\
\text { cantilever } 1\end{array}$} & $d_{31}(\mathrm{pm} / \mathrm{V})$ & 5.1 & 6.0 & 7.2 & 7.1 \\
\hline & Resonance (kHz) & 18.2 & 18.0 & 18.3 & 18.3 \\
\hline & Q factor (in air) & 66 & 65 & 67 & 67 \\
\hline \multirow{3}{*}{$\begin{array}{c}\text { Long } \\
\text { cantilever } 2\end{array}$} & $d_{31}(\mathrm{pm} / \mathrm{V})$ & 5.00 & 5.27 & $\mathrm{NE}^{*}$ & 6.7 \\
\hline & Resonance (kHz) & 19.5 & 19.1 & $\mathrm{NM}^{* *}$ & 19.1 \\
\hline & Q factor (in air) & 67 & 71 & $\mathrm{NE}$ & 69 \\
\hline \multirow{3}{*}{$\begin{array}{c}\text { Medium } \\
\text { cantilever } 1\end{array}$} & $d_{31}(\mathrm{pm} / \mathrm{V})$ & 1.2 & 9.0 & 14.2 & 13.9 \\
\hline & Resonance (kHz) & 466.6 & 467.4 & 473.2 & 472.9 \\
\hline & Q factor (in air) & 208 & 185 & 223 & 228 \\
\hline \multirow{3}{*}{$\begin{array}{c}\text { Medium } \\
\text { cantilever } 2\end{array}$} & $d_{31}(\mathrm{pm} / \mathrm{V})$ & 3.1 & 6.9 & 10.9 & $\mathrm{NE}$ \\
\hline & Resonance $(\mathrm{kHz})$ & 443.5 & 442.0 & 441.4 & NM \\
\hline & Q factor (in air) & 109 & 100 & 100 & $\mathrm{NE}$ \\
\hline \multirow{3}{*}{$\begin{array}{c}\text { Medium } \\
\text { cantilever } 3\end{array}$} & $d_{31}(\mathrm{pm} / \mathrm{V})$ & & 5.7 & 10.8 & $\mathrm{NE}$ \\
\hline & Resonance (kHz) & undetectable & 501.0 & 504.7 & NM \\
\hline & Q factor (in air) & & 108 & 103 & $\mathrm{NE}$ \\
\hline \multirow{3}{*}{$\begin{array}{c}\text { Short } \\
\text { cantilever } 1\end{array}$} & $d_{31}(\mathrm{pm} / \mathrm{V})$ & & $\mathrm{NE}$ & 11.5 & 10.9 \\
\hline & Resonance (MHz) & undetectable & NM & 5.72 & 5.71 \\
\hline & Q factor (in air) & & $\mathrm{NE}$ & 381 & 382 \\
\hline \multirow{3}{*}{$\begin{array}{c}\text { Short } \\
\text { cantilever } 2\end{array}$} & $d_{31}(\mathrm{pm} / \mathrm{V})$ & & $\mathrm{NE}$ & 12.8 & $\mathrm{NE}$ \\
\hline & Resonance (MHz) & undetectable & NM & 5.14 & NM \\
\hline & Q factor (in air) & & $\mathrm{NE}$ & 92 & $\mathrm{NE}$ \\
\hline \multirow{3}{*}{$\begin{array}{c}\text { Short } \\
\text { cantilever } 3\end{array}$} & $d_{31}(\mathrm{pm} / \mathrm{V})$ & & $\mathrm{NE}$ & 8.6 & $\mathrm{NE}$ \\
\hline & Resonance (MHz) & undetectable & NM & 3.57 & NM \\
\hline & Q factor (in air) & & $\mathrm{NE}$ & 34 & $\mathrm{NE}$ \\
\hline
\end{tabular}

*Not Estimated, **Not Measured

Table IV also indicates the changes in resonance frequencies, $d_{31}$ values and quality factors as a function of poling time for long, medium size and short cantilevers. As mentioned in section III. B, poling treatment might lead to either changes in the mechanical characteristics of the PZT films or additional beam curvature. In general, the resonance frequencies of the cantilevers decrease after the first 15 minutes of poling, but after further poling, the resonance frequencies tend to increase along with the $d_{31}$ values.

\section{E. Validation with mechanical frequency response measurements}


To validate the $d_{31}$ estimation method, the tip deflection of the cantilevers was measured using a Polytec MSA-500 laser vibrometer. The cantilevers were excited with a voltage signal (a periodic chirp) applied to the PZT and the velocity of several points on the cantilevers were measured. Figure 13 illustrates the deformed shape of a $150 \mu \mathrm{m}$ long cantilever at its first three resonances, showing excellent qualitative agreement with the theoretical mode shapes of a cantilever beam. Figure 14(a) gives the deformed shape of a $35 \mu \mathrm{m}$ medium cantilever around its fundamental resonance. To provide more quantitative insight, a detailed view of the anchor with magnified amplitude is also given in Fig. 14(b), showing that, as suspected in section IV. A, the clamping of the cantilever is not perfect. Figures 14(c) and (d) show that the ratio between the maximum amplitude to the amplitude at the theoretical clamping zone $(x=0)$ is very small, of the order of $1 \%$. Similar results for non-ideal clamping have been observed in a reference. ${ }^{36}$

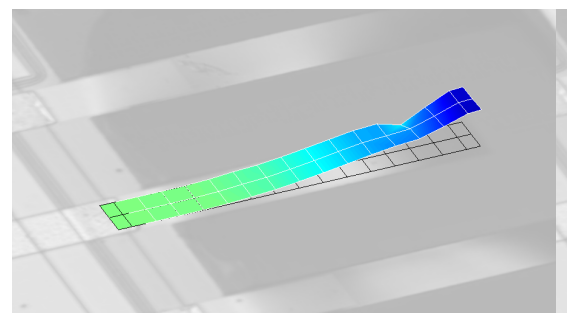

$18.34 \mathrm{kHz}$

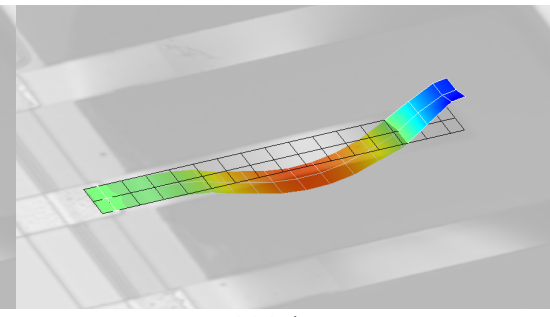

$102 \mathrm{kHz}$

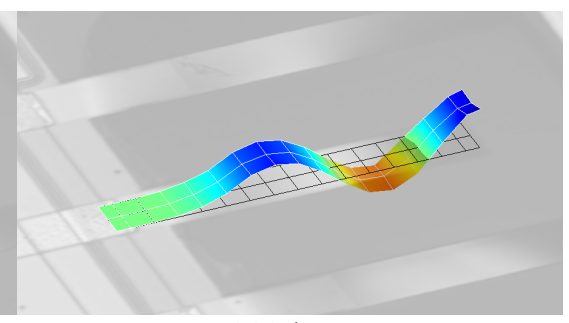

$292 \mathrm{kHz}$

FIG. 13. First three bending mode shapes of a $150 \mu \mathrm{m}$ long cantilever measured with the laser vibrometer.

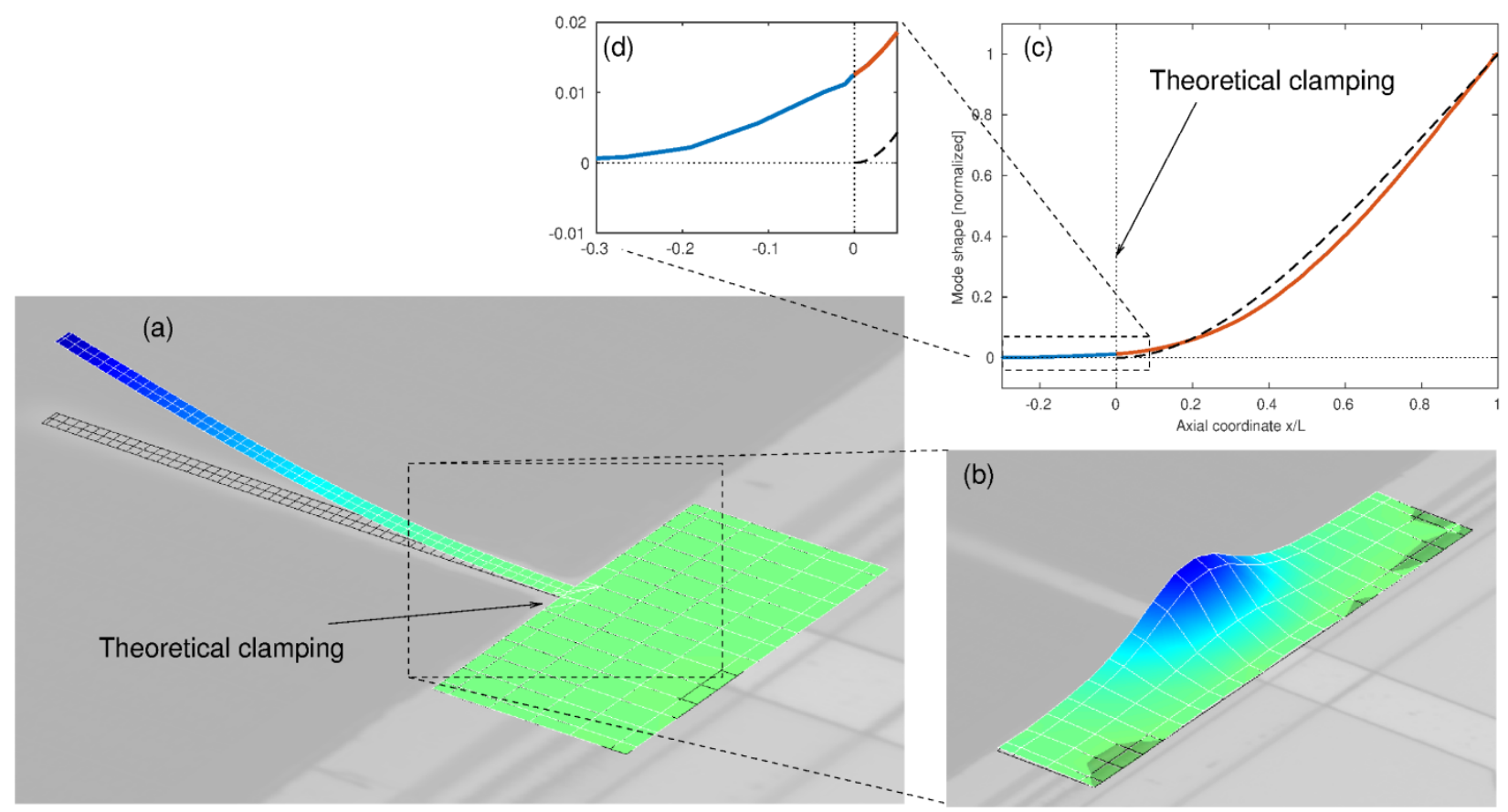

FIG. 14. First bending mode shape of a $35 \mu \mathrm{m}$ long medium cantilever measured with the laser vibrometer at $477.2 \mathrm{kHz}$. (a) 3D view and (b) zoom on the anchor with magnified amplitude, (c) measured profile (solid line) taken from the 3D view, theoretical deformed shape (dashed line) and (d) zoom in at the anchor area. 
The laser was then focused on the tip of a cantilever and the frequency response $H_{v}(\Omega)$ was determined from the measured velocity signal (obtained by fast Fourier transforms and averaged over time) divided by the input voltage. The results are identical to those obtained with a network analyzer and homodyne detection. Figure 15 shows the tip frequency response of a medium cantilever (with its fundamental resonance around $500 \mathrm{kHz}$ ) and of a long cantilever (with its first three resonance peaks). The short cantilevers frequency response was not measured since the maximum detection frequency of the laser vibrometer was $2 \mathrm{MHz}$, greater than their fundamental frequency.

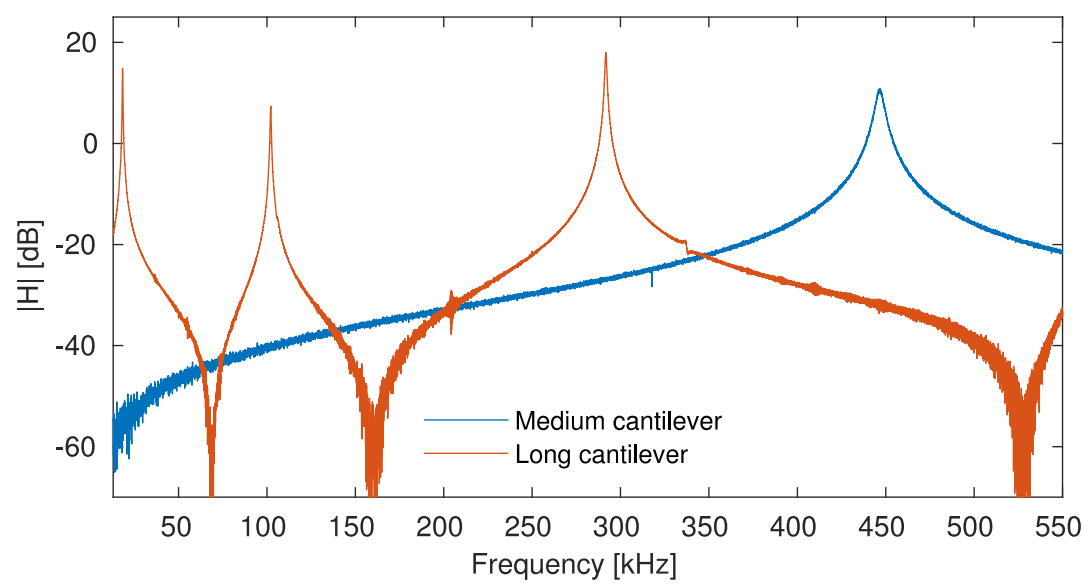

FIG. 15. Experimental frequency response functions: tip displacement of the cantilevers for a unit voltage in the PZT layer as a function of driving frequency, measured by the laser vibrometer.

From the equivalent single layer theory described in the Appendix, the displacement amplitude at the tip of the cantilever $w_{\mathrm{t} 0}$, at resonance, is:

$$
H\left(\omega_{k}\right) \approx \frac{w_{\mathrm{t} 0}}{V_{0}}=\gamma d_{31} \quad \text { with } \quad \gamma=\frac{Q_{k} Y_{p} \hat{\Theta} \Phi_{k}(1) \Phi_{k}^{\prime}(1)}{m L^{2} \omega_{k}^{2}}
$$

which allows the piezoelectric constant to be estimated as:

$$
d_{31}=\frac{H\left(\omega_{k}\right)}{\gamma}
$$

Around a given resonance, the natural frequency $\omega_{k}$, the Q-factor and the amplitude of $H_{v}\left(\omega_{k}\right)=\omega_{k} H\left(\omega_{k}\right)$ ( $H_{v}$ is the velocity frequency response, whereas $H$ is in displacement) were determined.

Table $\mathrm{V}$ shows the estimation of the $d_{31}$ piezoelectric constant obtained from the motional capacitance and the laser vibrometer (mechanical response). The two methods are reasonably close. The measurements of this section were performed about 12 months after those of section IV. D. The obtained values are a little smaller, probably due to aging of the PZT layers. 
TABLE V. Estimated values of the $d_{31}$ piezoelectric constant with the motional capacitance method and the mechanical frequency response method for two cantilevers of Table IV.

\begin{tabular}{|l|c|c|c|}
\hline & \multicolumn{2}{|c|}{ Long cantilever 1 } & Medium cantilever 2 \\
\hline & Mode 1 & Mode 3 & Mode 1 \\
\hline Natural frequency [kHz] & 18.3 & 291.6 & 446.8 \\
\hline Quality factor & 67 & 298 & 223 \\
\hline $\begin{array}{l}d_{31} \text { motional capacitance } \\
\text { estimation [pm/V] }\end{array}$ & 7.2 & 8.3 & 10.9 \\
\hline $\begin{array}{l}d_{31} \text { mechanical response } \\
\text { estimation [pm/V] }\end{array}$ & 8.7 & 7.8 & 6.9 \\
\hline
\end{tabular}

\section{CONCLUSIONS}

Silicon based cantilevers integrating $281 \mathrm{~nm}$ thick microcontact printed $\mathrm{PbZr}_{0.3} \mathrm{Ti}_{0.7} \mathrm{O}_{3}$ films were fabricated. The resonance frequencies of the cantilevers were detected via the piezoelectric response of the integrated PZT films, thus validating the capability of simultaneous excitation and detection of the PZT cantilevers even at small sizes (e.g. $10 \mu \mathrm{m}$-long and $2 \mu \mathrm{m}$-wide cantilevers had a resonance frequency of $5.7 \mathrm{MHz}$ and a Q factor of 381 in air). By analytical modelling of the PZT cantilevers and validations of the modelling by finite element simulation, $d_{31}$ was estimated to be around $10 \mathrm{pm} / \mathrm{V}$. Thin PZT films for realization of NEMS have been used for integrated actuation and detection on a geometrically complex elastic structure. This estimation of $d_{31}$ piezoelectric coefficient was consistent with values obtained from the mechanical response measured by a laser vibrometer. Hence, microcontact printed PZT films with poling treatment offer promising transduction schemes for piezoelectric NEMS with integrated actuation and sensing capabilities.

\section{ACKNOWLEDGMENTS}

The French General Delegation for Armament and the French National Agency for Research (grant ANR/PNANO 2008, project NEMSPIEZO 'ANR-08-NANO-015'), and a National Security Science and Engineering Faculty Fellowship are gratefully acknowledged for financial support. Cédric Ayela and Isabelle Dufour from IMS Bordeaux as well as Dolores Manrique from LAAS Toulouse are acknowledged for technical support and open access to the laser vibrometer.

This work was supported by LAAS-CNRS technology platform, a member of Renatech network. 


\section{Appendix: single layer equivalent theory}

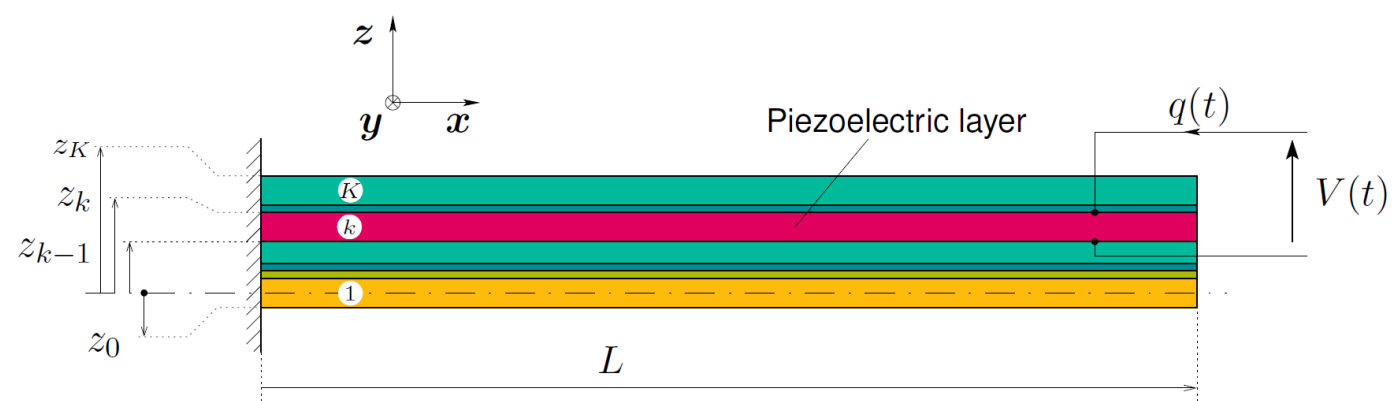

Figure A.1 Sketch of the laminated cantilever beam composed of $K$ layers.

As per the theory, ${ }^{30}$ a laminated beam composed of $K \in \mathbb{N}$ elastic layers is considered, where one layer is piezoelectric (Fig. A.1). The beam is clamped at $x=0$ and free at $x=L, x$ being the axial coordinate. The $k^{\text {th }}$ layer $(k=1, \ldots K)$ is situated between heights $z_{k-1}$ and $z_{k}, z$ being the bending direction; its thickness is $h_{k}=z_{k}-z_{k-1}$ and its width (in the $y$ direction) is $b_{k}$. A beam with a uniform cross section over its whole length $L$ is considered. This allows evaluation of an equivalent single layer theory for the beam deformations, with the plane cross section remaining plane and orthogonal to the middle line (EulerBernoulli assumptions or classical theory of laminate composites ${ }^{34}$ ). The deformation of the beam in bending along $z$ is characterized by the transverse displacement field along this direction $w(x, t)$ at time $\mathrm{t}$ and axial position $\mathrm{x}$, which is coupled to the voltage difference $V(t)$ between the electrodes of the piezoelectric layer and the electric charge $q(t)$ contained in its upper electrode. With this theory, $w(x, t)$ obeys the following equations: ${ }^{30}$

$$
\left\{\begin{array}{c}
\widehat{D} w_{, x x x x}+m \ddot{w}+\widehat{\Theta}[\delta(x-L)]_{, x}=0, \quad \forall x \in\left[\begin{array}{ll}
0 & L
\end{array}\right] \\
q-\hat{C} V-\left.\widehat{\Theta} w_{, x}\right|_{x=L}=0,
\end{array}\right.
$$

where $\widehat{D}$ is the bending stiffness of the beam, $m$ is its mass per unit length, $\widehat{\Theta}$ is the piezoelectric coupling coefficient, $\hat{C}$ is the blocked electric capacitance of the piezoelectric layer, $\delta(x)$ is the Dirac delta function, $O_{, x}=\partial() / \partial x$ and $\ddot{O}=\partial() / \partial t$. The electromechanical characteristics of the beam $m, \widehat{D}, \widehat{\Theta}, \hat{C}$ as a function of the geometrical/material properties of the layers are defined by:

$$
\widehat{D}=D-\frac{B^{2}}{A}, \quad \widehat{\Theta}=\Theta-\frac{B}{A} \Xi, \quad \hat{C}=C+\frac{\Xi^{2}}{A L},
$$

with:

$$
m=\sum_{k=1}^{K} b_{k} h_{k} \rho_{k}, \quad A=\sum_{k=1}^{K} b_{k} h_{k} Y_{k}, \quad B=\sum_{k=1}^{K} b_{k} \frac{z_{k}^{2}-z_{k-1}^{2}}{2} Y_{k}, \quad D=\sum_{k=1}^{K} b_{k} \frac{z_{k}^{3}-z_{k-1}^{3}}{2} Y_{k},
$$




$$
\Xi=b_{p} Y_{p} d_{31}, \quad \Theta=b_{p} \frac{z_{p}+z_{p-1}}{2} Y_{p} d_{31}, \quad C=\epsilon_{33} \frac{b_{p} L}{h_{p}}
$$

where $Y_{k}$ and $\rho_{k}$ are the Young's modulus and the mass density of the $k^{\text {th }}$ layer, $p$ is the index of the piezoelectric layer and $d_{31}$ and $\epsilon_{33}$ are its piezoelectric and dielectric constants. $D, A, B$ are respectively the bending, axial and axial/bending stiffness parameters of the beam; $\Theta, \Xi$ are respectively the bending and axial piezoelectric parameters of the beam. The modified parameters that appear in Eqs. (A.1), denoted with a hat $(\widehat{D}, \widehat{\Theta}, \hat{C})$, are independent of the vertical reference $z=0$ of the lamination, which can be chosen arbitrarily. It is also possible to choose the vertical reference so that $B=0$, which in this case leads to $\widehat{D}=$ $D, \widehat{\Theta}=\Theta$ and $\hat{C}=C$. The reader can refer to a reference ${ }^{30}$ for more details.

The eigenmodes of the beam in short circuit $(V=0)$ are defined as follows. The natural frequencies, in $[\mathrm{Hz}]$, can be computed from:

$$
f_{k}=\frac{\omega_{k}}{2 \pi}, \text { with } \omega_{k}=\frac{\beta_{k}^{2}}{L^{2}} \sqrt{\frac{\widehat{D}}{m}}
$$

and the mode shapes are:

$$
\Phi_{k}(\bar{x})=\cos \beta_{k} \bar{x}-\cosh \beta_{k} \bar{x}+\frac{\sin \beta_{k}-\sinh \beta_{k}}{\cos \beta_{k}+\cosh \beta_{k}}\left(\sin \beta_{k} \bar{x}-\sinh \beta_{k} \bar{x}\right),
$$

with $\bar{x}=x / L . \beta_{k}$ is the $k^{\text {th }}$ solution of:

$$
\frac{1}{\cosh \beta}+\cos \beta=0
$$

because of the clamped - free boundary conditions, defined by:

$$
\Phi_{k}(0)=0, \quad \Phi_{k}^{\prime}(0)=0, \quad \Phi_{k}^{\prime \prime}(1)=0, \quad \Phi_{k}^{\prime \prime \prime}(1)=0,
$$

where ()$^{\prime}=\mathrm{d}() / \mathrm{d} \bar{x}$. With Eq. (A.6), the mode shapes are naturally normalized:

$$
\int_{0}^{1} \Phi_{k}^{2}(\bar{x}) \mathrm{d} \bar{x}=1
$$

With the above equations, the frequency parameters, the mode shape amplitude at the tip (the free end) and the slope at the same location are:

$$
\begin{aligned}
\beta_{1}=1.8751, \quad \beta_{2}= & 4.6941, \quad \beta_{3}=7.8548, \quad \beta_{4}=10.996 \\
& \Phi_{k}^{\prime}(1)=2, \quad \forall k, \\
\Phi_{1}^{\prime}(1)=2.7530, \quad \Phi_{2}^{\prime}(1)= & 9.5616, \quad \Phi_{3}^{\prime}(1)=15.697, \quad \Phi_{4}^{\prime}(1)=21.990,
\end{aligned}
$$

The equations of motion (A.1) are now expanded on the $k^{\text {th }}$ eigenmode: 


$$
w(x, t)=\Phi_{k}(x) \eta(t)
$$

where $\eta(t)$ is the modal coordinate. Thanks to the orthogonality of the modes, the problem can be written as:

$$
\left\{\begin{array}{c}
\ddot{\eta}+\frac{\omega_{k}}{Q_{k}} \dot{\eta}+\omega_{k}^{2} \eta-\frac{\chi_{k}}{m L} V=0 \\
q=\hat{C} V+\underbrace{\chi_{k} \eta}_{q_{\mathrm{mot}}}
\end{array}\right.
$$

with the modal piezoelectric coefficient defined by:

$$
\chi_{k}=\frac{\widehat{\Theta} \Phi_{k}^{\prime}(1)}{L}
$$

and where a linear modal damping term has been added, of quality factor $Q_{k}$. The motional electric charge $q_{\text {mot }}$, defined in Eq. (A.12), is the part of the electric charge generated by the piezoelectric coupling.

For the frequency response of the cantilever beam, if a sine voltage $V(t)=V_{0} \cos \Omega t$ is applied across the piezoelectric layer, the modal coordinate and the motional electric charge are sine functions, that are written $\eta(t)=\operatorname{Re}(\hat{\eta} \exp j \Omega t)=\eta_{0} \cos (\Omega t+\varphi)$ and $q_{\mathrm{mot}}(t)=\operatorname{Re}\left(\hat{q}_{\mathrm{mot}} \exp j \Omega t\right)=q_{0} \cos (\Omega t+\psi)$. With Eq. (A.12), the complex amplitudes $\hat{\eta}=\eta_{0} \exp j \varphi$ and $\hat{q}_{\text {mot }}=q_{0} \exp j \psi$ can be written:

$$
\hat{\eta}(\Omega)=\frac{\chi_{k}}{m L} \frac{1}{\omega_{k}^{2}-\Omega^{2}+2 j \omega_{k} \Omega / Q_{k}} V_{0}, \quad \hat{q}_{\mathrm{mot}}(\Omega)=\chi_{k} \hat{\eta}(\Omega)
$$

Both $\hat{\eta}(\Omega)$ and $\hat{q}_{\text {mot }}(\Omega)$ display a resonant response as a function of $\Omega$.

The response of the cantilever at the resonance frequency, $\Omega \approx \omega_{k}$, is:

$$
\eta_{0}=\frac{\chi_{k} Q_{k}}{m L \omega_{k}^{2}} V_{0}, \quad q_{0=} \frac{\chi_{k}^{2} Q_{k}}{m L \omega_{k}^{2}} V_{0}
$$

Considering the one-mode approximation of Eq. (A.11) and Eq. (A.13), the amplitude of the tip displacement at the $k^{\text {th }}$ resonance can be written:

$$
w_{\mathrm{t} 0}=\Phi_{k}(1) \eta_{0}=\frac{Q_{k} \widehat{\Theta} \Phi_{k}(1) \Phi_{k}^{\prime}(1)}{m L^{2} \omega_{k}^{2}} V_{0} .
$$

The motional capacitance, defined by the ratio of $q_{0}$ and $V_{0}$ at resonance, ${ }^{31}$ is then:

$$
C_{\mathrm{mot}}=\frac{Q_{k} \widehat{\Theta}^{2} \Phi_{k}^{\prime 2}(1)}{m L^{3} \omega_{k}^{2}}
$$


Comparing Eqs. (A.2) and (A.4) shows that $\widehat{\Theta}$ is proportional to $Y_{p} d_{31}$ :

$$
\widehat{\Theta}=\widehat{\Theta} Y_{p} d_{31},
$$

with $\widehat{\widehat{\Theta}}$ of dimension $\left[\mathrm{m}^{2}\right]$. As a consequence, Eqs. (A.16)-(A.17) can be rewritten:

$$
\frac{w_{\mathrm{t} 0}}{V_{0}}=\underbrace{\frac{Q_{k} Y_{p} \widehat{\hat{\Theta}} \Phi_{k}(1) \Phi_{k}^{\prime}(1)}{m L^{2} \omega_{k}^{2}}}_{\gamma} d_{31}, \quad C_{\mathrm{mot}}=\underbrace{\frac{Q_{k} Y_{p}^{2} \widehat{\bar{\Theta}}^{2} \Phi_{k}^{\prime 2}(1)}{m L^{3} \omega_{k}^{2}}}_{\alpha} d_{31}^{2}
$$

The parameters $\gamma$ (dimensionless) and $\alpha$ (homogeneous to $[\mathrm{N} / \mathrm{m}]$ ), depend on the geometrical and material properties of the beam (through parameters $Y_{p}, \widehat{\widehat{\Theta}}, m$ and $L$ ) and its $k^{\text {th }}$ eigenmode $\left(\omega_{k}, \Phi_{k}, Q_{k}\right)$.

\section{REFERENCES}

${ }^{1}$ H. G. Craighead, "Nanoelectromechanical systems," Science 290, 1532-1535 (2000).

2 M. L. Roukes, "Nanoelectromechanical systems face the future," Phys. World 14, 25-31 (2001).

${ }^{3}$ K. L. Ekinci, "Electromechanical transducers at the nanoscale: actuation and sensing of motion in nanoelectromechanical systems (NEMS)," Small Journal 1, 786-797 (2005).

${ }^{4}$ Y. T. Yang, C. Callegari, X. L. Feng, K. L. Ekinci, and M. L. Roukes, "Zeptogram-scale nanomechanical mass sensing," Nano Letters 6, 583-586 (2006).

5 J. Chaste, A. Eichler, J. Moser, G. Ceballos, R. Rurali, and A. Bachtold, "A nanomechanical mass sensor with yoctogram resolution," Nat. Nanotech. 7, 301-304 (2012).

6 J. L. Arlett, E. B. Myers, and M. L. Roukes, "Comparative advantages of mechanical biosensors," Nature Nanotechnology 6. 203-215 (2011).

${ }^{7}$ B. Ilic, Y. Yang, K. Aubin, R. Reichenbach, S. Krylov, and H. G. Craighead, "Enumeration of DNA molecules bound to a nanomechanical oscillator," Nano Letters 5, 925-929 (2005).

${ }^{8}$ Z. J. Davis, G. Abadal, B. Helbo, O. Hansen, F. Campabadal, F. Pérez-Murano, J. Esteve, E. Figueras, J. Verd, N. Barniol, and A. Boisen, "Monolithic integration of mass sensing nano-cantilevers with CMOS circuitry," Sensors and Actuators A 105, 311-319 (2003).

9 R. B. Karabalin, M. H. Matheny, X. L. Feng, E. Defaÿ, G. Le Rhun, C. Marcoux, S. Hentz, P. Andreucci, and M. L. Roukes, "Piezoelectric nanoelectromechanical resonators based on aluminum nitride thin films," Appl. Phys. Lett. 95, 1031112009.

${ }^{10}$ N. Sinha, G. E. Wabiszewski, R. Mahameed, V. V. Felmetsger, S. M. Tanner, R. W. Carpick, and G. Piazza, "Piezoelectric aluminum nitride nanoelectromechanical actuators," Appl. Phys. Lett. 95, 053106 (2009).

${ }^{11}$ Z. Qian, F. Liu, Y. Hui, S. Kar, and M. Rinaldi, "Graphene as a massless electrode for ultrahigh-frequency piezoelectric nanoelectromechanical systems," Nano Lett. 15, 4599-4604 (2015).

${ }^{12} \mathrm{~L}$. Nicu and T. Leïchlé, "Biosensors and tools for surface functionalization from the macro- to the nanoscale: the way forward," J. Appl. Phys. 104, 111101 (2008).

${ }^{13}$ S. Trolier-McKinstry and P. Muralt, "Thin film piezoelectrics for MEMS," J. Electroceram. 12, 7-17 (2004).

${ }^{14}$ C. Lee, T. Itoh, T. Ohashi, R. Maeda, and T. Suga, "Development of a piezoelectric self-excitation and self-detection mechanism in PZT microcantilevers for dynamic scanning force microscopy in liquid," Journal of Vacuum Science \& Technology B 15, 1559-1563 (1997). 
${ }^{15}$ T. Itoh, T. Ohashi, and T. Suga, "Piezoelectric cantilever array for multiprobe scanning force microscopy," IEEE Int. Conference MEMS 1996, 451-455 (1996).

${ }^{16}$ F. Mathieu, F. Larramendy, D. Dezest, C. Huang, G. Lavallee, S. Miller, C.M. Eichfeld, W. Mansfield, S. Trolier-McKinstry, and L. Nicu, "Reducing parasitic effects of actuation and sensing schemes for piezoelectric microelectromechanical resonators," Microelec. Eng. 111, 68-76 (2013).

${ }^{17}$ S. Guillon, D. Saya, L. Mazenq, J. Costecalde, D. Remiens, C. Soyer, and L. Nicu, "Lead zirconate titanate nanoscale patterning by ultraviolet-based lithography lift-off. technique for nanoelectromechanical system applications," IEEE Trans. Ultrason. Ferroelectr. Freq. Control 59, 1955-1961 (2012).

${ }^{18}$ D. Dezest, O. Thomas, F. Mathieu, L. Mazenq, C. Soyer, J. Costecalde, D. Remiens, J.-F. Deü, and L. Nicu, "Wafer-scale fabrication of self-actuated piezoelectric nanoelectromechanical resonators based on lead zirconate titanate (PZT)," J. Microelec. Microeng. 25, 35002-35013 (2015).

${ }^{19}$ A. J. Welsh, R. H.T. Wilke, M. A. Hickner, S. Trolier-McKinstry, "Low-cost, damage-free patterning of lead zirconate titanate films," J. Am. Ceram. Soc. 96, 2799-2805 (2013).

${ }^{20}$ D. Saya, D. Dezest, A.J. Welsh, O. Thomas, F. Mathieu, T. Leichle, L. Nicu and S. Trolier-McKinstry, "Fabrication and characterization of mechanical resonators integrating microcontact printed PZT films", 2017 Joint IEEE-International Symposium on the Applications of Ferroelectric (ISAF)/International Workshop on Acoustic Transduction Materials and Devices (IWATMD)/Piezoresponse Force Microscopy (PFM), May 7-11, 2017, Atlanta, USA.

${ }^{21}$ G. Arndt, E. Defay, G. Le Rhun, P. Rey, F. Perruchot, M. Aid, L. Liu, and M. Miller, "Dynamic analytical modelling and piezoelectric characterization of a $\mathrm{Pb}(\mathrm{Zr}, \mathrm{Ti}) \mathrm{O}_{3} / \mathrm{SiN}$ cantilever with losses," J. Micromech. Microeng. 20, 115019 (2010).

${ }^{22} \mathrm{H}$. G. Yeo, and S. Trolier-McKinstry, "Effect of piezoelectric layer thickness and poling conditions on the performance of cantilever piezoelectric energy harvesters on Ni foils," Sensors and Actuators A 273, 90-97 (2018).

${ }^{23}$ J. F. Shepard Jr, P. J. Moses, and S. Trolier-McKinstry, “The wafer flexure technique for the determination of the transverse piezoelectric coefficient $\left(\mathrm{d}_{31}\right)$ of PZT thin films," Sens. \& Act. A 71, 133-138 (1998).

${ }^{24}$ E. M. A. Fuentes-Fernandez, B. E. Gnade, M. A. Quevedo-Lopez, P. Shah, and H. N. Alshareef "The effect of poling conditions on the performance of piezoelectric energy harvesters fabricated by wet chemistry," J. Mater. Chem. A 3, 9837-9842 (2015).

${ }^{25}$ A. Lebied, B. Necib, and M. Sahli, "Analytical modeling of a piezoelectric bimorph beam", American Journal of Mechanical Engineering 4, 7-10 (2016).

${ }^{26}$ L. H. Kang, D. O. Lee, and J.-H. Han, "A measurement method for piezoelectric material properties under longitudinal compressive stress-a compression test method for thin piezoelectric materials," Measurement Science and Technology 22, 065701 (2011).

${ }^{27}$ C. M. Costa, V. Sencadas, J. F. Mano, and S. Lanceros-Méndez, "Effect of poling on the mechanical properties of $\beta$-poly(vinylidene fluoride)," Materials Science Forum 514-516, 951-955 (2006).

${ }^{28}$ J. F. Shepard. F. Chu, I. Kanno, and S. Trolier-McKinstry, "Characterization and aging response of the d31 piezoelectric coefficient of lead zirconate titanate thin films," Journal of Applied Physics 85, 67116716 (1999).

${ }^{29}$ A. Kholkin, E. Colla, K. Brooks, P. Muralt, M. Kohli, T. Maeder, D. Taylor, and N. Setter, "Interferometric study of piezoelectric degradation in ferroelectric thin films," Microelectronic Engineering 29, 261-264 (1995).

${ }^{30}$ J. Ducarne, O. Thomas, and J. F. Deü, "Placement and dimension optimization of shunted piezoelectric patches for vibration reduction," J. Sound Vib. 331, 3286-3303 (2012).

${ }^{31} \mathrm{O}$. Thomas, B. Legrand, and C. Fuinel, "Optimization of length and thickness of smart transduction layers on beam structures for control and M/NEMS applications," SMASIS 2015 Colorado Springs, USA, Sept. $2015,8857$. 
${ }^{32}$ D. Das, L. Sanchez, J. Martin, B. Power, S. Isaacson, R. G. Polcawich, and I. Chasiotis, "Control of mechanical response of freestanding $\mathrm{PbZr}_{0.52} \mathrm{Ti}_{0.48} \mathrm{O}_{3}$ films through texture," Appl. Phys. Lett. 109, 131905 (2016).

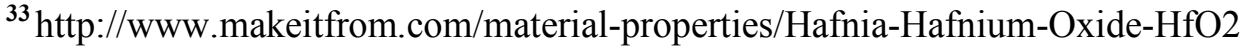

${ }^{34}$ O. Thomas, J.-F. Deü, and J. Ducarne, "Vibration of an elastic structure with shunted piezoelectric patches: efficient finite-element formulation and electromechanical coupling coefficients," International Journal of Numerical Methods in Engineering 80, 235-268 (2009).

${ }^{35} \mathrm{H}$. Miki, K. Kushida-Abdelghafar, K. Torii, and Y. Fujisaki, "Hydrogen-related degradation and recovery phenomena in $\mathrm{Pb}(\mathrm{Zr}, \mathrm{Ti}) \mathrm{O}_{3}$ capacitors with a platinum electrode," Japanese Journal of Applied Physics 36,1132-1135 (1997).

${ }^{36}$ J.N. Reddy, "Mechanics of laminated composite plates and shells: theory and analysis," CRC Press, (1997). 